\title{
UCRL-TR-229427
}

LAWRENCE LIVERMORE N A T IO N A L LABORATORY

R. Soltz, R. Gupta

March 27, 2007 
This document was prepared as an account of work sponsored by an agency of the United States Government. Neither the United States Government nor the University of California nor any of their employees, makes any warranty, express or implied, or assumes any legal liability or responsibility for the accuracy, completeness, or usefulness of any information, apparatus, product, or process disclosed, or represents that its use would not infringe privately owned rights. Reference herein to any specific commercial product, process, or service by trade name, trademark, manufacturer, or otherwise, does not necessarily constitute or imply its endorsement, recommendation, or favoring by the United States Government or the University of California. The views and opinions of authors expressed herein do not necessarily state or reflect those of the United States Government or the University of California, and shall not be used for advertising or product endorsement purposes.

This work was performed under the auspices of the U.S. Department of Energy by University of California, Lawrence Livermore National Laboratory under Contract W-7405-Eng-48. 


\section{Lattice QCD Thermodynamics First 5000 Trajectories}

\section{Background}

These results represent the first LQCD analysis for approximately 5000 trajectories with each of the p4rhmc and milc codes, with some of the lower temperature runs having fewer. Both runs were for lattice dimensions of $32^{3} x 8$. Some $32^{4} \mathrm{~T}=0$ jobs were also run for $\mathrm{p} 4 \mathrm{rhmc}$. The $\mathrm{p} 4$ calculation was performed with v2.0 QMP_MPI.X (semi-optomized p4 code using qmp over mpi) and milc version of the su3_rhmc_susc_eos executable dated Mar 1, 2007 on ubgl in the /usr/gapps/hip/qcd/milc/bin subdirectory (svn revision 28). As with previous runs, calculations were performed along lines of constant physics, with the light quark masses 2-3 times their physics values and the strange quark mass set by $m_{u d}=0.1 m_{s}$.

Job submissions were performed using a new subSet.pl job submission script that locates current jobs and submits additional jobs with the same beta value as pending. Note that after reaching a limit of about 35 jobs subsequent submissions are delayed and will not be submitted directly from that state. The job submission script was used to submit revised versions of the milc and p4rhmc csh scripts. Initial thermalized lattices for each code were also for milc (taken from the firstPhys runs), but the p4rhmc runs include thermalization. The only modifications for running on BG/L were to the directory names and the mT parameter which determines job durations ( $24 \mathrm{hrs}$ on BG/L vs. $4 \mathrm{hrs}$ on ubgl). All finite temperature jobs were submitted to the 512 node partitions, and all $\mathrm{T}=0$ runs were submitted to 2048 node partitions.

The set of runs was plagued by filesystem errors on lscratch1 and lscratch2. Many jobs had to be reset (deleting the most recent output file for milc and/or lattice for $\mathrm{p} 4$ ) and resubmitted. The analysis was performed with a new set of scripts that produced a more condensed output for scanning. All scans were verified with checksums, which have been retained in the output along with the line numbers. All lattices, log files, and job submission scripts have been archived to permanent storage in the 5k subdirectory for subsequent analysis. 


\section{MILC Results}

1: 2912: beta ns nt mud ms u0 dt accept $N$

2: $2432: 6.45803280 .00820 \quad 0.0820 \quad 0.85490 .01430 \quad 0.77 \quad 1775$

3: $2444: 6.500032 \quad 8 \quad 0.00765 \quad 0.0765 \quad 0.85690 .01430 \quad 0.793975$

4: $2428: 6.550032 \quad 8 \quad 0.00705 \quad 0.0705 \quad 0.8594 \quad 0.01430 \quad 0.794355$

5: $2408: 6.600032 \quad 8 \quad 0.00650 \quad 0.0650 \quad 0.8616 \quad 0.01430 \quad 0.804345$

6: $2441: 6.650032 \quad 8 \quad 0.00599 \quad 0.0599 \quad 0.8636 \quad 0.01430 \quad 0.814355$

7: $2423: 6.700032 \quad 8 \quad 0.00552 \quad 0.0552 \quad 0.8657 \quad 0.01250 \quad 0.841981$

8: $2407: 6.760032 \quad 8 \quad 0.00500 \quad 0.0500 \quad 0.8678 \quad 0.01250 \quad 0.854021$

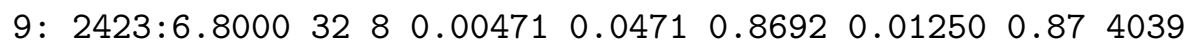

10: $2438: 6.850032 \quad 8 \quad 0.00437 \quad 0.0437 \quad 0.8709 \quad 0.01250 \quad 0.873785$

11: $2428: 6.900032 \quad 8 \quad 0.00407 \quad 0.0407 \quad 0.8726 \quad 0.01250 \quad 0.873849$

12: $2419: 6.950032 \quad 8 \quad 0.00380 \quad 0.03800 .8741 \quad 0.01250 \quad 0.884350$

13: $2424: 7.000032 \quad 8 \quad 0.00355 \quad 0.0355 \quad 0.8756 \quad 0.01250 \quad 0.894546$

14: $2413: 7.08003280 .00310 \quad 0.0310 \quad 0.87790 .01250 \quad 0.894126$

15: 4679: beta ns nt ReP dReP chi_L dchi_L cgiters dcgiters N

16: $2318: 6.458032 \quad 8 \quad 0.00230 .00010 .057 \quad 0.002 \quad 0.0 \quad 0.01775$

17: $2306: 6.500032 \quad 8 \quad 0.0033 \quad 0.00010 .061 \quad 0.002 \quad 0.0 \quad 0.03975$

18: $2311: 6.550032 \quad 8 \quad 0.00490 .0001 \quad 0.061 \quad 0.002 \quad 0.0 \quad 0.04355$

19: $2317: 6.600032 \quad 8 \quad 0.00790 .0001 \quad 0.0690 .002 \quad 0.0 \quad 0.04345$

20: $2309: 6.650032 \quad 8 \quad 0.0123 \quad 0.0002 \quad 0.072 \quad 0.003 \quad 0.0 \quad 0.04355$

21: $2327: 6.700032 \quad 8 \quad 0.01890 .0003 \quad 0.078 \quad 0.004 \quad 0.0 \quad 0.01981$

22: $2315: 6.76003280 .0273 \quad 0.0004 \quad 0.086 \quad 0.004 \quad 0.0 \quad 0.04021$

23: $2317: 6.800032 \quad 8 \quad 0.0337 \quad 0.0003 \quad 0.093 \quad 0.004 \quad 0.0 \quad 0.04039$

24: $2338: 6.85003280 .03990 .0003 \quad 0.086 \quad 0.003 \quad 0.0 \quad 0.03785$

25: $2336: 6.900032 \quad 8 \quad 0.0467 \quad 0.0003 \quad 0.0890 .005 \quad 0.0 \quad 0.03849$

26: $2315: 6.950032 \quad 8 \quad 0.0531 \quad 0.0003 \quad 0.094 \quad 0.003 \quad 0.0 \quad 0.04350$

27: $2319: 7.000032 \quad 8 \quad 0.0588 \quad 0.0003 \quad 0.092 \quad 0.003 \quad 0.0 \quad 0.04546$

28: $2304: 7.08003280 .0701 \quad 0.0004 \quad 0.104 \quad 0.004 \quad 0.0 \quad 0.04126$

29: 4881: beta ns nt pbp_ud dpbp_udpbp_s dpbp_s plaq dplaq $\mathrm{N}$

30: $3364: 6.458032 \quad 8 \quad 0.0793640 .000140 \quad 0.193556 \quad 0.0000891 .6026200 .0000541775$

31: $3343: 6.500032 \quad 8 \quad 0.066863 \quad 0.000110 \quad 0.175463 \quad 0.000071 \quad 1.6182200 .0000443975$

32: $3329: 6.550032 \quad 8 \quad 0.053600 \quad 0.000120 \quad 0.155673 \quad 0.000070 \quad 1.636270 \quad 0.0000414355$

33: $3334: 6.600032 \quad 8 \quad 0.0420590 .000120 \quad 0.1378920 .000070 \quad 1.653130 \quad 0.0000394345$

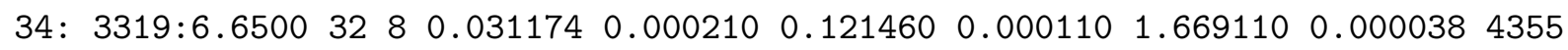

35: $3318: 6.700032 \quad 8 \quad 0.020816 \quad 0.000240 \quad 0.1062030 .0001201 .684630 \quad 0.000041 \quad 1981$

36: $3316: 6.760032 \quad 8 \quad 0.013420 \quad 0.000180 \quad 0.090604 \quad 0.000140 \quad 1.701890 \quad 0.0000514021$

37: $3333: 6.8000 \quad 32 \quad 8 \quad 0.010618 \quad 0.000088 \quad 0.082050 \quad 0.000097 \quad 1.712920 \quad 0.0000304039$

38: $3365: 6.850032 \quad 8 \quad 0.008477 \quad 0.0000390 .0728450 .000063 \quad 1.7263600 .000037 \quad 3785$

39: $3334: 6.900032 \quad 8 \quad 0.007124 \quad 0.0000190 .065141 \quad 0.000046 \quad 1.739310 \quad 0.000032 \quad 3849$

$40: 3343: 6.950032 \quad 8 \quad 0.006224 \quad 0.000023 \quad 0.0586990 .000054 \quad 1.751810 \quad 0.0000264350$

41: $3314: 7.000032 \quad 8 \quad 0.005508 \quad 0.000010 \quad 0.053122 \quad 0.000033 \quad 1.763950 \quad 0.0000224546$

42: $3329: 7.080032 \quad 8 \quad 0.004520 \quad 0.000008 \quad 0.044391 \quad 0.000025 \quad 1.782670 \quad 0.0000264126$

43: 5697: beta ns nt rect drect pgm dpgm pb_dmdu_p_ud dpb_dmdu_p_ud $N$

44: $3331: 6.45803280 .842593 \quad 0.0000710 .8624790 .000083 \quad-4.893340 .000161775$

45: $3324: 6.500032 \quad 8 \quad 0.8623920 .000060 \quad 0.883637 \quad 0.000070 \quad-4.88978 \quad 0.000123975$

46: $3304: 6.550032 \quad 8 \quad 0.885542 \quad 0.000055 \quad 0.908170 \quad 0.000060 \quad-4.880840 .000114355$

47: $3301: 6.600032 \quad 8 \quad 0.907245 \quad 0.000051 \quad 0.931090 \quad 0.000067 \quad-4.873890 .000114345$

48: $3329: 6.650032 \quad 8 \quad 0.927926 \quad 0.000055 \quad 0.9528790 .000061 \quad-4.868180 .00013 \quad 4355$ 
49: $3310: 6.700032 \quad 8 \quad 0.948170 \quad 0.000061 \quad 0.974103 \quad 0.000067 \quad-4.85857 \quad 0.00013 \quad 1981$ $50: 3305: 6.760032 \quad 8 \quad 0.970673 \quad 0.000075 \quad 0.997600 \quad 0.000081 \quad-4.85143 \quad 0.000154021$ 51: $3287: 6.800032 \quad 8 \quad 0.985080 \quad 0.000041 \quad 1.012640 \quad 0.000057 \quad-4.845130 .000074039$ 52: $3286: 6.85003281 .002690 \quad 0.000052 \quad 1.030940 \quad 0.000057 \quad-4.83712 \quad 0.000103785$ 53: $3307: 6.900032 \quad 8 \quad 1.019700 \quad 0.000048 \quad 1.048640 \quad 0.000045 \quad-4.828590 .00006 \quad 3849$ 54: $3289: 6.95003281 .0361600 .0000391 .065750 \quad 0.000036 \quad-4.821180 .000074350$ 55: $3268: 7.00003281 .052230 \quad 0.0000291 .0823800 .000033 \quad-4.81350 \quad 0.000054546$ 56: $3263: 7.08003281 .077090 \quad 0.000041 \quad 1.108110 \quad 0.000042 \quad-4.80150 \quad 0.000064126$ 57: 7663: beta ns nt pb_dmdu_p_s dpb_dmdu_p_s chl_pbp_d dchl_pbp_d chl_pbp_c dchl_pbp_c N 58: $3109: 6.458032 \quad 8 \quad-4.839890 .00018 \quad 0.52250 \quad 0.06080 \quad 1.02650 \quad 0.002001775$ 59: $3083: 6.500032 \quad 8 \quad-4.84434 \quad 0.00015 \quad 0.56050 \quad 0.04090 \quad 1.04170 \quad 0.001003975$ $60: 3087: 6.550032 \quad 8 \quad-4.84323 \quad 0.00010 \quad 0.59140 \quad 0.03540 \quad 1.06280 \quad 0.001804355$ 61: $3086: 6.600032 \quad 8 \quad-4.84295 \quad 0.00011 \quad 0.63190 \quad 0.05040 \quad 1.09260 \quad 0.00240 \quad 4345$ 62: $3102: 6.650032 \quad 8 \quad-4.84294 \quad 0.00015 \quad 0.97190 \quad 0.14440 \quad 1.14210 \quad 0.002904355$ 63: $3094: 6.700032 \quad 8 \quad-4.83813 \quad 0.000120 .63180 \quad 0.06810 \quad 1.19440 \quad 0.003501981$

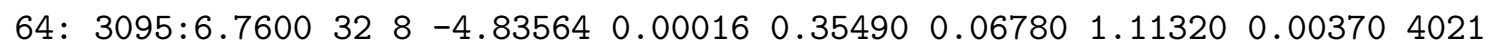
65: $3085: 6.800032 \quad 8 \quad-4.83176 \quad 0.000090 .18440 \quad 0.01650 \quad 1.02140 \quad 0.003504039$ 66: $3087: 6.850032 \quad 8 \quad-4.82621 \quad 0.00007 \quad 0.06000 \quad 0.00400 \quad 0.92770 \quad 0.002603785$ 67: $3097: 6.900032 \quad 8 \quad-4.81949 \quad 0.00007 \quad 0.02640 \quad 0.00200 \quad 0.856600 .001403849$ 68: $3080: 6.950032 \quad 8 \quad-4.81346 \quad 0.00007 \quad 0.01500 \quad 0.00240 \quad 0.80890 \quad 0.002404350$ 69: $3070: 7.000032 \quad 8 \quad-4.80702 \quad 0.00005 \quad 0.00660 \quad 0.00080 \quad 0.77090 \quad 0.001204546$ $70: 3076: 7.0800328 \quad-4.796850 .00006 \quad 0.00330 \quad 0.00110 \quad 0.72670 \quad 0.001004126$ 71: 7501: beta ns nt Chl_pbp_t dchl_pbp_t Chl_pbp_s dchl_pbp_s Chl_pbps_d dchl_pbps_d N 72: $3069: 6.45803281 .54900 \quad 0.06020 \quad 2.57550 \quad 0.05970 \quad 0.21590 \quad 0.024401775$ 73: $3044: 6.500032881 .60210 \quad 0.04070 \quad 2.64380 \quad 0.04040 \quad 0.21770 \quad 0.018403975$ 74: $3066: 6.55003281 .65410 \quad 0.03560 \quad 2.71690 \quad 0.03590 \quad 0.18960 \quad 0.013504355$

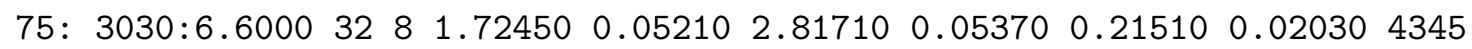
76: $3051: 6.650032 \quad 8 \quad 2.11400 \quad 0.14540 \quad 3.25610 \quad 0.14650 \quad 0.22980 \quad 0.04460 \quad 4355$ 77: $3063: 6.700032 \quad 8 \quad 1.82620 \quad 0.06870 \quad 3.02060 \quad 0.06940 \quad 0.18950 \quad 0.019101981$ 78: $3062: 6.760032 \quad 8 \quad 1.46810 \quad 0.06940 \quad 2.58120 \quad 0.07130 \quad 0.17990 \quad 0.02760 \quad 4021$ 79: $3047: 6.800032 \quad 8 \quad 1.20580 \quad 0.01730 \quad 2.22720 \quad 0.01870 \quad 0.15730 \quad 0.01690 \quad 4039$

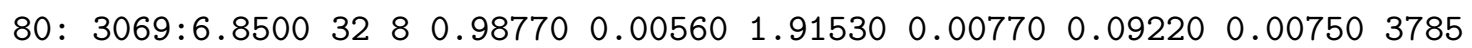

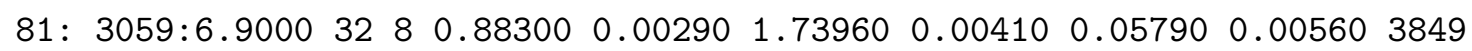
82: $3035: 6.950032 \quad 8 \quad 0.82400 \quad 0.00470 \quad 1.63290 \quad 0.00700 \quad 0.04860 \quad 0.005004350$ 83: $3042: 7.000032 \quad 8 \quad 0.77740 \quad 0.00170 \quad 1.54830 \quad 0.00290 \quad 0.02480 \quad 0.001904546$ 84: $3035: 7.080032 \quad 8 \quad 0.72990 \quad 0.00170 \quad 1.45660 \quad 0.00250 \quad 0.01410 \quad 0.001904126$ 85: 7959: beta ns nt Chl_pbps_c dchl_pbps_c Chl_pbps_t dchl_pbps_t Chl_pbp_ss dchl_pbp_ss N 86: $3049: 6.458032 \quad 8 \quad 0.64030 \quad 0.00030 \quad 0.85620 \quad 0.024301 .49650 \quad 0.024201775$ 87: $3060: 6.500032 \quad 8 \quad 0.66010 \quad 0.00020 \quad 0.87780 \quad 0.01850 \quad 1.53790 \quad 0.018503975$ 88: $3037: 6.550032 \quad 8 \quad 0.68060 \quad 0.00030 \quad 0.87020 \quad 0.01350 \quad 1.55080 \quad 0.013504355$ 89: $3030: 6.600032 \quad 8 \quad 0.69830 \quad 0.00030 \quad 0.91340 \quad 0.02040 \quad 1.61170 \quad 0.020504345$ $90: 3052: 6.650032 \quad 8 \quad 0.71570 \quad 0.00050 \quad 0.94550 \quad 0.04490 \quad 1.66120 \quad 0.045104355$ 91: $3048: 6.700032 \quad 8 \quad 0.73430 \quad 0.00070 \quad 0.92390 \quad 0.01900 \quad 1.65820 \quad 0.019001981$ 92: $3057: 6.760032 \quad 8 \quad 0.74860 \quad 0.00070 \quad 0.92850 \quad 0.02720 \quad 1.67700 \quad 0.026804021$ 93: $3040: 6.800032 \quad 8 \quad 0.75210 \quad 0.00040 \quad 0.90940 \quad 0.01690 \quad 1.66140 \quad 0.01700 \quad 4039$ 94: $3049: 6.850032 \quad 8 \quad 0.75010 \quad 0.00030 \quad 0.84230 \quad 0.00760 \quad 1.59240 \quad 0.007603785$ 95: $3047: 6.900032 \quad 8 \quad 0.74290 \quad 0.00040 \quad 0.80080 \quad 0.00550 \quad 1.54370 \quad 0.005403849$ 96: $3038: 6.950032 \quad 8 \quad 0.73190 \quad 0.00020 \quad 0.78040 \quad 0.00490 \quad 1.51230 \quad 0.004904350$ 97: $3043: 7.0000 \quad 32 \quad 8 \quad 0.71990 \quad 0.00020 \quad 0.74470 \quad 0.00180 \quad 1.46470 \quad 0.001804546$ 98: $3014: 7.080032 \quad 8 \quad 0.70030 \quad 0.00020 \quad 0.71440 \quad 0.00190 \quad 1.41480 \quad 0.002104126$ 
99: 6393: beta ns nt chl_II dchl_II Chl_Oud dchl_Oud Chl_S dchl_S Chl_BB dchl_BB N 100: $3437: 6.458032 \quad 8 \quad 0.05890 .0026 \quad 0.12930 .4416 \quad 0.03330 .01920 .0557 \quad 0.0768 \quad 1775$

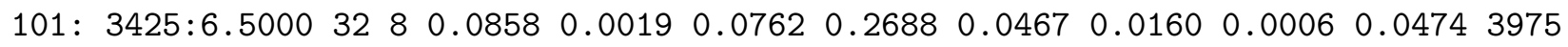

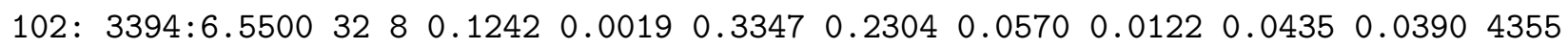
103: $3391: 6.600032 \quad 8 \quad 0.1818 \quad 0.0032 \quad 0.7501 \quad 0.1600 \quad 0.1344 \quad 0.01090 .12800 .02824345$ 104: $3419: 6.650032 \quad 8 \quad 0.2579 \quad 0.0032 \quad 0.8474 \quad 0.1600 \quad 0.2490 \quad 0.0109 \quad 0.1664 \quad 0.0307 \quad 4355$ 105: $3423: 6.700032 \quad 8 \quad 0.3482 \quad 0.0038 \quad 1.0899 \quad 0.1472 \quad 0.3590 \quad 0.0141 \quad 0.2413 \quad 0.0275 \quad 1981$

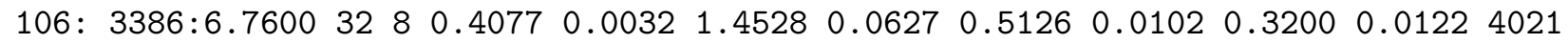
107: $3406: 6.800032 \quad 8 \quad 0.43390 .0026 \quad 1.6723 \quad 0.0512 \quad 0.6106 \quad 0.01280 .36290 .01024039$

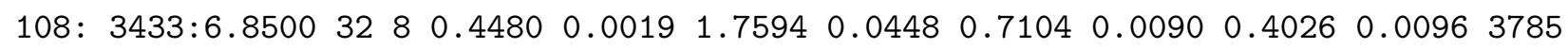
109: $3430: 6.900032 \quad 8 \quad 0.4576 \quad 0.0013 \quad 1.7920 \quad 0.03580 .7610 \quad 0.0083 \quad 0.41920 .0077 \quad 3849$ 110: $3424: 6.950032 \quad 8 \quad 0.4627 \quad 0.0013 \quad 1.7690 \quad 0.0314 \quad 0.8096 \quad 0.0077 \quad 0.42820 .0064 \quad 4350$ 111: $3422: 7.000032 \quad 8 \quad 0.46590 .0006 \quad 1.8656 \quad 0.0326 \quad 0.8346 \quad 0.0070 \quad 0.44800 .0064 \quad 4546$ 112: $3432: 7.080032 \quad 8 \quad 0.4742 \quad 0.0013 \quad 1.8618 \quad 0.0294 \quad 0.8870 \quad 0.0064 \quad 0.4595 \quad 0.0058 \quad 4126$ 113: 4725: beta ns nt chi_YY dchi_YY chi_VB dchi_YB 0_11 d0_11 N

114: $3105: 6.4580328 \quad-0.0070 \quad 0.0461 \quad-0.00256 \quad 0.04992 \quad-0.02688 \quad 0.108801775$

115: $2997: 6.500032 \quad 8 \quad 0.0416 \quad 0.0256 \quad 0.00448 \quad 0.03072 \quad-0.06656 \quad 0.070403975$ 116: $2989: 6.550032 \quad 8 \quad 0.06850 .0230 \quad 0.03200 \quad 0.02688 \quad-0.04032 \quad 0.057604355$ 117: $2968: 6.600032 \quad 8 \quad 0.1267 \quad 0.0179 \quad 0.06016 \quad 0.01792 \quad 0.00576 \quad 0.039684345$ 118: $3009: 6.650032 \quad 8 \quad 0.1696 \quad 0.0147 \quad 0.04416 \quad 0.01664 \quad-0.04608 \quad 0.038404355$ 119: $3004: 6.700032 \quad 8 \quad 0.20990 .0160 \quad 0.04160 \quad 0.01728 \quad-0.07552 \quad 0.03648 \quad 1981$ 120: $3008: 6.76003280 .2995 \quad 0.0077 \quad 0.04992 \quad 0.00768 \quad-0.04416 \quad 0.016004021$ 121: $2990: 6.800032 \quad 8 \quad 0.3610 \quad 0.0077 \quad 0.05632 \quad 0.00576 \quad-0.01536 \quad 0.012804039$ 122: $2994: 6.85003280 .3923 \quad 0.0064 \quad 0.04032 \quad 0.00576 \quad-0.00832 \quad 0.011523785$ 123: $2999: 6.900032 \quad 8 \quad 0.4096 \quad 0.0051 \quad 0.03200 \quad 0.00448 \quad-0.00960 \quad 0.008963849$ 124: $2998: 6.950032 \quad 8 \quad 0.4192 \quad 0.0051 \quad 0.01728 \quad 0.00384 \quad-0.02048 \quad 0.007684350$ 125: $2933: 7.000032 \quad 8 \quad 0.4358 \quad 0.0045 \quad 0.02240 \quad 0.00448 \quad 0.00064 \quad 0.008324546$ 126: $3015: 7.08003280 .4493 \quad 0.0045 \quad 0.00896 \quad 0.00384 \quad-0.00896 \quad 0.007684126$ 127: 4659: beta ns nt 0_ss d0_ss 0_ls d0_1s C_ss dC_ss dSI ddSI N 128: $3779: 6.458032 \quad 8 \quad-0.0064 \quad 0.0192 \quad 0.04290 .0301 \quad 0.0397 \quad 0.0006 \quad-1.30 e-02 \quad 1.40 e-02 \quad 1775$ 129: $3819: 6.5000328-0.01150 .0160 \quad-0.0294 \quad 0.0186 \quad 0.0582 \quad 0.0013 \quad-2.55 e-03 \quad 1.10 e-02 \quad 3975$ 130: $3833: 6.5500328-0.0358 \quad 0.0115 \quad-0.0326 \quad 0.0160 \quad 0.0928 \quad 0.0019-4.26 e-03 \quad 8.20 e-034355$ 131: $3782: 6.6000328-0.01920 .0109-0.0294 \quad 0.0141 \quad 0.1536 \quad 0.0026 \quad 6.32 e-04 \quad 7.60 e-034345$ 132: $3735: 6.65003280 .0051 \quad 0.0109 \quad-0.0243 \quad 0.0141 \quad 0.2438 \quad 0.0038 \quad 9.30 e-04 \quad 7.40 e-034355$ 133: $3727: 6.700032 \quad 8 \quad-0.0243 \quad 0.0141 \quad 0.0000 \quad 0.0160 \quad 0.3827 \quad 0.0058 \quad 1.80 e-02 \quad 7.70 e-03 \quad 1981$ 134: $3768: 6.760032 \quad 8 \quad-0.0198 \quad 0.0102-0.0115 \quad 0.0077 \quad 0.5325 \quad 0.0070 \quad 1.42 \mathrm{e}-03 \quad 7.10 \mathrm{e}-034021$ 135: $3827: 6.800032 \quad 8 \quad-0.0141 \quad 0.0115 \quad-0.02690 .0096 \quad 0.6246 \quad 0.0051 \quad-5.12 \mathrm{e}-03 \quad 5.70 \mathrm{e}-03 \quad 4039$ 136: $3745: 6.850032 \quad 8 \quad 0.0070 \quad 0.0083 \quad-0.0134 \quad 0.0064 \quad 0.7034 \quad 0.0038 \quad 2.28 e-03 \quad 4.80 e-03 \quad 3785$ 


\section{P4RHMC Results}

\section{P4RHMC therm 200}

1: $772: \mathrm{p} 4 \mathrm{~b} 3.4610200$

$\begin{array}{lllll}2: & 3241: \mathrm{hmc} 1190 & 2.28373717815602 & 0.984500662798978 & 0.551609342143434\end{array}$

3: $559:$ pbp 2380

4: $5310: 3.1300000000000001 \mathrm{e}-02 \quad 0.1547770727528590 .0001395655585304181 .75528227862555 \mathrm{e}-06 \quad 3.0579$

5: $5227: 3.1300000000000000 \mathrm{e}-03 \quad 0.072509561571813 \quad 0.000214207044442213 .28965745242429 \mathrm{e}-06 \quad 7.13170$

6: 4880:plaq $1190 \quad 0.5241271078558632 .63239285929818 \mathrm{e}-05 \quad 1.02630092094242 \mathrm{e}-07 \quad 1.25006262553322 \mathrm{e}-0$

7: 4913:rect $1190 \quad 0.284086514782008 \quad 3.61890368987989 \mathrm{e}-05 \quad 1.78625324171321 \mathrm{e}-07 \quad 2.17132217666611 \mathrm{e}-0$

8: 5057 :wline $47600.004770809298905350 .000517133006954068 \quad 8.01432019122566 \mathrm{e}-05 \quad 5.41721256485592$

9: $775: \mathrm{p} 4 \mathrm{~b} 3.4910200$

10: $3071:$ hmc 27101.821331358381891 .062373569500620 .6338760577251

11: $557:$ pbp 5420

12: $5418: 2.8999999999999998 \mathrm{e}-03 \quad 0.0514360667397930 .0001429223780403014 .24390722615055 \mathrm{e}-06 \quad 6.950$

13: $5413: 2.9000000000000001 \mathrm{e}-02 \quad 0.130782205297505 \quad 8.57867676777658 \mathrm{e}-051.71168630191889 \mathrm{e}-062.698$

14: 4908:plaq $2700 \quad 0.531122685754042 \quad 1.52292184339761 \mathrm{e}-05 \quad 9.36413976260209 \mathrm{e}-08 \quad 7.65996118152651 \mathrm{e}-\mathrm{c}$

15: 4878:rect $2700 \quad 0.292728754722416 \quad 2.10445976604369 \mathrm{e}-05 \quad 1.6680485329279 \mathrm{e}-07 \quad 1.49269321691389 \mathrm{e}-0$

16: 5140:wline $108800.009095031873351160 .0003170857337651898 .43965377493829 \mathrm{e}-05 \quad 3.247512405178 \mathrm{e}$

17: $768: p 4 b 3.5110200$

18: $3235:$ hmc $41101.68205220657853 \quad 0.994007727215251 \quad 0.593649353953963$

19: $556: \mathrm{pbp} 8200$

$20: 5351: 2.5899999999999999 \mathrm{e}-02 \quad 0.113053828433919 \quad 7.50413724218205 \mathrm{e}-05 \quad 2.085707617201 \mathrm{e}-06 \quad 2.77073$

21: $5507: 2.5899999999999999 \mathrm{e}-03 \quad 0.0399906915876792 \quad 0.0001362715529115796 .52002281118241 \mathrm{e}-06 \quad 8.588$

22: 4870 : plaq $4100 \quad 0.53534962303888 \quad 1.24392081774682 \mathrm{e}-05 \quad 9.38904657892942 \mathrm{e}-08 \quad 5.63144562801235 \mathrm{e}-0$

23: 4932 : rect $4100 \quad 0.297942476861794 \quad 1.77971688825512 \mathrm{e}-05 \quad 1.78193176198009 \mathrm{e}-07 \quad 1.20796300042849 \mathrm{e}-\mathrm{c}$

24: 5083:wline $163600.01178597157710530 .0002833129902513479 .39536669135908 \mathrm{e}-05 \quad 3.06809515680378$

25: $771: \mathrm{p} 4 \mathrm{~b} 3.5410200$

26: $3193: \mathrm{hmc} 40801.296697948650621 .000280297157890 .627002386552576$

27: $561: \mathrm{pbp} 8160$

28: 5463:2.3999999999999998e-03 $0.0216060135269103 \quad 0.000140129890722848 \quad 7.33063118441672 \mathrm{e}-064.94$ 29: 5369:2.4000000000000000e-02 $0.0932624178254974 \quad 7.87764673034942 \mathrm{e}-05 \quad 2.3345708780525 \mathrm{e}-06 \quad 1.753$ 30: 4901 :plaq $4080 \quad 0.541530409486011 \quad 1.26591216654537 \mathrm{e}-05 \quad 9.81380142505803 \mathrm{e}-08 \quad 4.95090878107549 \mathrm{e}-\mathrm{c}$ 31: 4933:rect $4070 \quad 0.305640360675882 \quad 1.78239207278186 \mathrm{e}-05 \quad 1.83883924908681 \mathrm{e}-07 \quad 9.35388780254322 \mathrm{e}-\mathrm{c}$ 32: 5000:wline $162400.02296545437383290 .000308440170209806 \quad 0.000101374935719046 \quad 3.3971409458902$ 33: $774:$ p4b3.57 10200

34: $3215: \mathrm{hmc} 34601.04787405896927 \quad 1.04055463095274 \quad 0.692479369634969$

35: $563: \mathrm{pbp} 6920$

36: 5506:2.1199999999999999e-03 $0.0110721341287027 \quad 7.08505022690926 \mathrm{e}-05 \quad 1.57499293223534 \mathrm{e}-06 \quad 1.14$ 37: 5415:2.1200000000000000e-02 $0.0742735543252463 \quad 6.93164987652464 \mathrm{e}-05 \quad 1.52162925251971 \mathrm{e}-06 \quad 9.90$ 38: 4879:plaq $34800.5472811489481331 .1266239714398 \mathrm{e}-05 \quad 7.86883407528611 \mathrm{e}-08 \quad 3.31617405847953 \mathrm{e}-0 \mathrm{~s}$ 39: 4911 : rect $3480 \quad 0.312812395073295 \quad 1.60465583026745 e-05 \quad 1.45326448293116 e-07 \quad 6.82277960442566 e-c$ 40: 4991:wline 138800.03383771148511720 .0003531513327730420 .0001126324618709884 .6696850209121 41: $768: p 4 b 3.6010200$

42: 3228:hmc $5070 \quad 0.9222451356387931 .019002049920810 .705702659572893$

43: $604:$ pbp 10180

$44: 5531: 1.9199999999999998 \mathrm{e}-02 \quad 0.0614216098474517 \quad 3.27272935841719 \mathrm{e}-05 \quad 5.00912905350844 \mathrm{e}-07 \quad 3.34$ 45: 5443:1.9200000000000000e-03 $0.007154183905655542 .18668006572306 \mathrm{e}-05 \quad 2.25389217761111 \mathrm{e}-072.5$ 46: 4916:plaq $50800.552523931794401 \quad 7.93823783740037 \mathrm{e}-06 \quad 6.75409155394746 \mathrm{e}-08 \quad 2.19923500167612 \mathrm{e}-\mathrm{c}$ 47: 4916:rect $50800.3193171804217991 .09605449074172 \mathrm{e}-05 \quad 1.22292755426996 \mathrm{e}-07 \quad 4.14348889347121 \mathrm{e}-\mathrm{c}$ 
48: 4942:wline $203200.042592212401998 \quad 0.000262480576539934 \quad 0.0001050494635102762 .93123703334978$ 49: $771: p 4 b 3.6310200$

50: $3240:$ hmc $6660 \quad 0.890024324874446 \quad 1.04090453146678 \quad 0.707195855317339$

51: $605: \mathrm{pbp} 13340$

52: $5487: 1.6999999999999999 \mathrm{e}-03 \quad 0.005396935530178851 .1197547377436 \mathrm{e}-05 \quad 7.8521002428067 \mathrm{e}-08 \quad 1.060$ 53: 5292:1.7000000000000001e-02 $0.05039820715032732 .5122223678201 \mathrm{e}-05 \quad 3.9612979544077 \mathrm{e}-072.2098$ 54: 4939:plaq $6650 \quad 0.557545489179279 \quad 7.60045597121762 \mathrm{e}-06 \quad 7.18154729049022 \mathrm{e}-08 \quad 2.37616371299571 \mathrm{e}-\mathrm{c}$ 55: 4890 :rect $6650 \quad 0.325567529555828 \quad 1.08310314251138 \mathrm{e}-05 \quad 1.35033410847375 \mathrm{e}-07 \quad 4.89115921360218 \mathrm{e}-\mathrm{c}$ 56: 4940:wline $26600 \quad 0.0513584200569410 .0002374732395378410 .0001101341455086542 .78467063606645$ 57: $774: \mathrm{p} 4 \mathrm{~b} 3.6610200$

58: $3239: \mathrm{hmc} 5870 \quad 0.829130572344353 \quad 1.01634035935656 \quad 0.699064762229276$

59: $611: \mathrm{pbp} 11780$

60: $5534: 1.6999999999999999 \mathrm{e}-03 \quad 0.0049164643691585 \quad 6.49119689408213 \mathrm{e}-06 \quad 2.30043172913184 \mathrm{e}-08 \quad 3.038$ 61: 5402:1.7000000000000001e-02 $0.04732603320978251 .87076650436219 \mathrm{e}-05 \quad 1.91699732279878 \mathrm{e}-07 \quad 1.173$ 62: 4932 : plaq $58800.5622967379762327 .95297757748043 e-06 \quad 7.01152404314382 \mathrm{e}-08 \quad 2.60398545430608 \mathrm{e}-($ 63: 4906:rect $5890 \quad 0.3314769208031651 .15384160328961 \mathrm{e}-051.36901024386448 \mathrm{e}-07 \quad 5.09466930705329 \mathrm{e}-($ 64: 5042:wline 235600.05976025118668690 .0002468842423918740 .0001031563724367712 .97846986522718 65: $777: \mathrm{p} 4 \mathrm{~b} 3.6910200$

66: $3222: \mathrm{hmc} 7210 \quad 0.803255986745504 \quad 1.01103032594437 \quad 0.699205930346646$

67: $605: \mathrm{pbp} 14420$

68: 5469:1.5000000000000000e-03 $0.004055074613851313 .61758954314678 \mathrm{e}-06 \quad 8.84304387884115 \mathrm{e}-09 \quad 1.98$ 69: 5545:1.4999999999999999e-02 $0.03974824796932181 .20780722461758 \mathrm{e}-05 \quad 9.80087466125935 \mathrm{e}-08$ 5.61" 70: 4915:plaq $7210 \quad 0.566917268386676 \quad 6.30195625521702 \mathrm{e}-06 \quad 6.20173831294813 \mathrm{e}-08 \quad 1.72537923030976 \mathrm{e}-\mathrm{c}$ 71: 4910:rect $7210 \quad 0.337254661780662 \quad 8.92722589764644 \mathrm{e}-06 \quad 1.16733610134774 \mathrm{e}-07 \quad 3.41375000767264 \mathrm{e}-\mathrm{c}$ 72: 5016:wline 287600.06753376664563960 .0002414551472828920 .0001129602007108532 .6474854641570 73: $775: \mathrm{p} 4 \mathrm{~b} 3.76 \quad 10200$

$74: 3183: \mathrm{hmc} 7440 \quad 0.751747628069241 .025113577782290 .723619646321822$

75: $610:$ pbp 14920

76: $5590: 1.3899999999999999 \mathrm{e}-02 \quad 0.0335654423438473 \quad 6.58939394518621 \mathrm{e}-06 \quad 2.98893971539193 \mathrm{e}-08 \quad 1.70$ 77: 5466:1.3900000000000000e-03 $0.003378926163499651 .20959241258916 \mathrm{e}-06 \quad 1.01888916086653 \mathrm{e}-09$ 1.3 78: 4921:plaq $7450 \quad 0.577119606770267 \quad 5.88067788005432 \mathrm{e}-06 \quad 5.95743810250343 \mathrm{e}-08 \quad 1.51528350840478 \mathrm{e}-$ 79: 4925 : rect $7470 \quad 0.350094405928046 \quad 8.46569746876088 \mathrm{e}-06 \quad 1.16112171509653 \mathrm{e}-07 \quad 2.98342438024745 \mathrm{e}-\mathrm{c}$ 80: 5010:wline $298800.08506532613730490 .0002492139575109980 .000119832347241604 \quad 3.1765225730136$ 81: $768:$ :t0b3.60 10200

82: $3179: \mathrm{hmc} 1170 \quad 0.75221752298838 \quad 1.030824221152920 .695799255556701$

83: $555: \mathrm{pbp} 2340$

84: 5376:1.9199999999999998e-02 $0.07117883553192 \quad 7.5507158332097 e-05 \quad 5.30512466325487 e-07 \quad 1.24029$ 85: $5387: 1.9200000000000000 \mathrm{e}-03 \quad 0.02244791569952680 .0001253280995294711 .37686620558643 e-06 \quad 3.45$ 86: 4902:plaq $1160 \quad 0.552175947425559 \quad 1.10755226781022 \mathrm{e}-05 \quad 2.16942422914812 \mathrm{e}-08 \quad 1.89062706657923 \mathrm{e}-($ 87: 4878:rect $1160 \quad 0.31875911329621 \quad 1.51809143117196 \mathrm{e}-05 \quad 3.83570646797099 \mathrm{e}-08 \quad 3.91185426449688 \mathrm{e}-0 \mathrm{~s}$ 88: 5104:wline $46806.23803007292929 \mathrm{e}-05 \quad 0.000432385144065159 .47202351777235 \mathrm{e}-05 \quad 6.4382833062136 \mathrm{~s}$ 89: $774:$ tob3.66 10200

90: $3228:$ hmc $1100 \quad 0.419194410873643 \quad 1.01917733011626 \quad 0.763885272577777$

91: $550:$ pbp 2200

92: $5501: 1.6999999999999999 \mathrm{e}-03 \quad 0.0140417957989537 \quad 0.000140469166082604 \quad 1.67309177833798 \mathrm{e}-06 \quad 3.10$ 93: 5469:1.7000000000000001e-02 $0.05549723586134027 .77919808171996 \mathrm{e}-05 \quad 5.28565249886424 \mathrm{e}-07 \quad 9.60$ 94: 4894:plaq $1100 \quad 0.5620962672234831 .10863763059734 \mathrm{e}-05 \quad 1.96223520907177 \mathrm{e}-08 \quad 1.75811564541229 \mathrm{e}-\mathrm{c}$ 95: 4840:rect $1100 \quad 0.331140561380034 \quad 1.6956163885431 \mathrm{e}-05 \quad 3.97769588319743 \mathrm{e}-08 \quad 4.02217821451802 \mathrm{e}-0$ 96: 5128:wline $4400-8.56495079405035 e-050.0003890383943490939 .281033910898 e-05 \quad 5.4196099338464$ 97: $775:$ tob3.76 10200 
98: $3292:$ hmc $1120 \quad 0.272532243959523 \quad 0.9785776345410290 .797430099891305$

99: $552: \operatorname{pbp} 2220$

100: $5576: 1.3899999999999999 \mathrm{e}-02 \quad 0.0385520686932971 \quad 5.85569689214383 \mathrm{e}-05 \quad 3.02723174894919 \mathrm{e}-07 \quad 4.7 \mathrm{~s}$ 101: 5337:1.3900000000000000e-03 $0.00694950520088004 \quad 0.0001153385078469721 .16174407340151 \mathrm{e}-06 \quad 1$. 102: 4908:plaq $1120 \quad 0.577044970740007 \quad 7.98533434508519 \mathrm{e}-06 \quad 1.50112665649243 \mathrm{e}-08 \quad 1.03563677297357 \mathrm{e}$ 103: 4846:rect $1120 \quad 0.349960203436981 \quad 1.22532423072337 \mathrm{e}-05 \quad 3.0398172698514 \mathrm{e}-08 \quad 2.29575202948635 \mathrm{e}-\mathrm{c}$ 104: 5169:wline $4480-0.0004440752329802790 .0003991224304908948 .56200719992258 \mathrm{e}-054.8615684942$

\section{P4RHMC therm 400}

1: $774:$ p4b3.46 10400

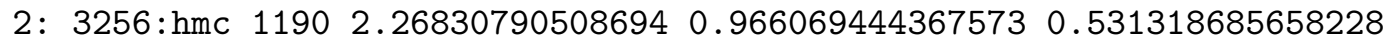

3: $559:$ pbp 2380

4: $5248: 3.1300000000000001 \mathrm{e}-02 \quad 0.154877031091864 \quad 0.000142329189746802 \quad 1.4293305904646 \mathrm{e}-06 \quad 2.70955$

5: $5375: 3.1300000000000000 \mathrm{e}-03 \quad 0.07272435057023890 .0002215448644949932 .59730893535062 \mathrm{e}-06 \quad 6.393$

6: 4912:plaq 11900.524108089157706 2.79615616024058e-05 $9.57104686777976 \mathrm{e}-08 \quad 1.21636060616116 \mathrm{e}-08$

7: 4967 :rect $1190 \quad 0.284059408964193 \quad 3.94808878789794 \mathrm{e}-05 \quad 1.71465205297918 \mathrm{e}-07 \quad 2.24396237549886 \mathrm{e}-0$

8: 5098:wline $47600.00468799507410354 \quad 0.000572707001076091 \quad 7.92499381629699 \mathrm{e}-056.11659149246776 \mathrm{e}$ 9: $777: \mathrm{p} 4 \mathrm{~b} 3.4910400$

10: $3171: \mathrm{hmc} 27101.853592090605181 .074237365737730 .602177015103896$

11: $557:$ pbp 5420

12: $5494: 2.8999999999999998 \mathrm{e}-03 \quad 0.0516226170612057 \quad 0.000132324694731675 \quad 3.15592878754395 e-06 \quad 4.049$ 13: $5372: 2.9000000000000001 \mathrm{e}-02 \quad 0.130909518968597 \quad 7.74481722310581 \mathrm{e}-051.25385757543248 \mathrm{e}-061.206$ 14: 4906:plaq $2700 \quad 0.5310984151472831 .42005647215898 \mathrm{e}-05 \quad 8.07000290795178 \mathrm{e}-08 \quad 4.09694715493035 \mathrm{e}-\mathrm{c}$ 15: 4890 :rect $2700 \quad 0.29269533878781 \quad 1.94769373694821 \mathrm{e}-05 \quad 1.41602430094698 \mathrm{e}-07 \quad 7.09612995325268 \mathrm{e}-0$ 16: 5077:wline $108800.00876834807709930 .0003306564452944828 .40906750733684 \mathrm{e}-05 \quad 3.4601434101947 \mathrm{~s}$ 17: $770: p 4 b 3.5110400$

18: $3209: \mathrm{hmc} 41101.692708158415230 .983686565876360 .571743658749326$

19: $556: \mathrm{pbp} 8200$

20: $5442: 2.5899999999999999 \mathrm{e}-02 \quad 0.113274255499035 \quad 5.75791613208669 \mathrm{e}-051.11774735044687 \mathrm{e}-068.663$ 21: $5444: 2.5899999999999999 \mathrm{e}-03 \quad 0.04037617801682710 .0001074012696592513 .53665223617006 \mathrm{e}-06 \quad 2.81$ 22: 4866 : plaq $4100 \quad 0.5353208269109081 .1039592070835 \mathrm{e}-05 \quad 7.81892680845908 \mathrm{e}-08 \quad 3.73606853875513 \mathrm{e}-0$ 23: 4934 : rect $4100 \quad 0.297898748051704 \quad 1.54521792780852 \mathrm{e}-05 \quad 1.41844832704068 \mathrm{e}-07 \quad 7.47673086227996 \mathrm{e}-\mathrm{c}$ 24: 5064:wline 163600.0114420177255774 0.000282914588723104 9.12744525569792e-05 3.07183590179403 25: $773: p 4 b 3.5410400$

26: $3187: \mathrm{hmc} 40801.29983974852126 \quad 0.98938535765126 \quad 0.610170712025543$

27: $561: \mathrm{pbp} 8160$

28: $5501: 2.3999999999999998 \mathrm{e}-03 \quad 0.0218409329705586 \quad 0.000135774006884327 \quad 6.49024774339672 \mathrm{e}-06 \quad 4.61$ 29: $5401: 2.4000000000000000 \mathrm{e}-02 \quad 0.0934485352173792 \quad 7.05591511395979 \mathrm{e}-05 \quad 1.75955384221464 \mathrm{e}-06 \quad 1.22$ 30: 4853:plaq $4080 \quad 0.5415064014521251 .1902790706655 \mathrm{e}-05 \quad 8.81547097196632 \mathrm{e}-08 \quad 4.10394909038576 \mathrm{e}-0$ 31: 4923:rect $4070 \quad 0.305605867099947 \quad 1.67426484057042 \mathrm{e}-05 \quad 1.63899216080953 \mathrm{e}-07 \quad 7.76096230166244 \mathrm{e}-\mathrm{c}$ 32: 5055:wline $162400.02263477521437160 .000309808005360193 \quad 9.97176042556564 \mathrm{e}-05 \quad 3.4472532692231$ 33: $776:$ p4b3. $57 \quad 10400$

34: $3180: \mathrm{hmc} 34601.05508827773737 \quad 1.04272054262724 \quad 0.672379982029412$

35: $563: \mathrm{pbp} 6920$

36: 5479:2.1199999999999999e-03 $0.0110810983057963 \quad 7.43752607125304 \mathrm{e}-05 \quad 1.63020081101726 \mathrm{e}-06 \quad 1.20$ 37: 5407:2.1200000000000000e-02 $0.0742949617173566 \quad 7.29333124330722 \mathrm{e}-05 \quad 1.58249284492638 \mathrm{e}-06 \quad 1.03$ 38: 4946:plaq $34800.5472757061595391 .15829004045278 \mathrm{e}-05 \quad 7.80279189970574 \mathrm{e}-08 \quad 3.38383095832794 \mathrm{e}-\mathrm{c}$ 39: 4904 :rect $34800.3128051650270861 .63955442723764 \mathrm{e}-05 \quad 1.43334036948512 \mathrm{e}-07 \quad 6.82510717480876 \mathrm{e}-\mathrm{c}$ 40: 5022:wline $138800.0340075819782278 \quad 0.000365309406927734 \quad 0.0001121303813456884 .85936525191528$ 
41: $770: p 4 b 3.6010400$

42: $3265:$ hmc $5070 \quad 0.928878016751397 \quad 1.01315159549878 \quad 0.693098919083509$

43: $604:$ pbp 10180

44: 5504:1.9199999999999998e-02 $0.0614187365646811 \quad 3.32934768729471 \mathrm{e}-05 \quad 4.9710789346817 \mathrm{e}-07 \quad 3.395$

45: $5394: 1.9200000000000000 \mathrm{e}-03 \quad 0.007148467556111942 .21458036433508 \mathrm{e}-05 \quad 2.2154780025482 \mathrm{e}-072.58$

46: 4960:plaq $5080 \quad 0.552523690935899 \quad 8.08658496516974 \mathrm{e}-06 \quad 6.74976448514528 \mathrm{e}-08 \quad 2.22927257167231 \mathrm{e}-($

47: 4862:rect $50800.319317263200021 \quad 1.11402927791873 \mathrm{e}-05 \quad 1.22101595246741 \mathrm{e}-07 \quad 4.16740644005942 \mathrm{e}-\mathrm{c}$

48: 4958:wline 203200.04250452438365450 .0002698603390070320 .0001056294070060063 .0411719675222

49: $773: \mathrm{p} 4 \mathrm{~b} 3.6310400$

50: $3228: \mathrm{hmc} 66600.893925223974467 \quad 1.040206308580740 .697841090311502$

51: $605: \mathrm{pbp} 13340$

52: $5561: 1.6999999999999999 \mathrm{e}-03 \quad 0.00539857053772747 \quad 1.14478429269957 \mathrm{e}-05 \quad 7.9534318110908 \mathrm{e}-081.09$

53: $5394: 1.7000000000000001 \mathrm{e}-02 \quad 0.05039873596242882 .56087460386463 \mathrm{e}-05 \quad 3.9895268973226 \mathrm{e}-072.263$

54: 4844:plaq 66500.5575429247389 7.76017110009963e-06 7.18714019736666e-08 2.43786473794575e-09

55: 4902:rect $6650 \quad 0.325564708813287 \quad 1.10698496162939 \mathrm{e}-05 \quad 1.35181768229309 \mathrm{e}-07 \quad 5.02701405240042 \mathrm{e}-$

56: $5017:$ wline $26600 \quad 0.05126188781574850 .0002403815602848990 .0001095349673711292 .81542342306178$

57: $776:$ p4b3.66 10400

58: $3247:$ hmc $5870 \quad 0.8383140046039651 .013923243869620 .688061645674588$

59: $611: \mathrm{pbp} 11780$

60: $5607: 1.6999999999999999 \mathrm{e}-03 \quad 0.00491963325575021 \quad 6.68041518063196 \mathrm{e}-06 \quad 2.34999806869709 \mathrm{e}-08$ 3.1

61: 5321:1.7000000000000001e-02 $0.04733689142351531 .90156807574774 \mathrm{e}-05 \quad 1.91001224225426 \mathrm{e}-07 \quad 1.18$

62: 4903:plaq $5880 \quad 0.562293110153143 \quad 8.09950086463117 \mathrm{e}-06 \quad 7.01212737393548 \mathrm{e}-08 \quad 2.66722431449185 \mathrm{e}-\mathrm{c}$

63: 4934:rect $5890 \quad 0.331471883464184 \quad 1.17484827574255 e-05 \quad 1.36461283838468 e-07 \quad 5.17543179023693 e-c$

64: 5030:wline 235600.0596343455977653 0.0002496066932863330 .0001022688570809332 .98904709401481

65: $779: \mathrm{p} 4 \mathrm{~b} 3.6910400$

66: $3231: \mathrm{hmc} 7210 \quad 0.808083600926078 \quad 1.00578261267192 \quad 0.690372036964756$

67: $605: \mathrm{pbp} 14420$

68: $5464: 1.5000000000000000 \mathrm{e}-03 \quad 0.00405402740368723 \quad 3.68612539471886 \mathrm{e}-06 \quad 8.91963575581785 \mathrm{e}-092.0$

69: 5456:1.4999999999999999e-02 $0.039742698187188 \quad 1.23025151956547 \mathrm{e}-05 \quad 9.88436600738647 \mathrm{e}-08 \quad 5.7910$

70: 4887 :plaq $7210 \quad 0.566915548477695 \quad 6.43032719772124 \mathrm{e}-06 \quad 6.19097515218406 \mathrm{e}-08 \quad 1.7603696882647 \mathrm{e}-05$

71: 4919:rect $7210 \quad 0.337253177589456 \quad 9.11987536729282 \mathrm{e}-06 \quad 1.16662167203062 \mathrm{e}-07 \quad 3.48803098209055 \mathrm{e}-\mathrm{c}$

72: 5049:wline $287600.06747878639464780 .0002455881982935790 .000112772177066252 \quad 2.7015341720809$ ?

73: $777: \mathrm{p} 4 \mathrm{~b} 3.7610400$

74: $3202: \mathrm{hmc} 7440 \quad 0.75713658840693 \quad 1.02337044865544 \quad 0.715767931728693$

75: $610:$ pbp 14920

76: 5563:1.3899999999999999e-02 $0.0335697051116886 \quad 6.65954088351637 \mathrm{e}-06 \quad 2.96716893685125 \mathrm{e}-08 \quad 1.72$

$77: 5456: 1.3900000000000000 \mathrm{e}-03 \quad 0.003379563694821691 .23227153235858 \mathrm{e}-06 \quad 1.02793313393956 \mathrm{e}-091.4$

78: 4956:plaq $7450 \quad 0.577117641576659 \quad 5.99797042672912 \mathrm{e}-06 \quad 5.96534774575754 \mathrm{e}-08 \quad 1.53922741055149 \mathrm{e}-($

79: 4911 : rect $7470 \quad 0.350091717734103 \quad 8.64198406712883 \mathrm{e}-06 \quad 1.16403478854768 \mathrm{e}-07 \quad 3.02732931734144 \mathrm{e}-$

80: $5026:$ wline $298800.08505379694068370 .000254906846370454 \quad 0.0001207762766013283 .2513808528920$

81: $770:$ :0b3.60 10400

82: $3315: \mathrm{hmc} 11700.742376383589032 \quad 0.984902936488737 \quad 0.688582343831169$

83: $555: \operatorname{pbp} 2340$

84: $5464: 1.9199999999999998 \mathrm{e}-02 \quad 0.0714589293360704 \quad 4.379186238986 \mathrm{e}-05 \quad 1.28937007909269 \mathrm{e}-072.0895$ 85: 5383:1.9200000000000000e-03 $0.0229255407151004 \quad 7.22943711927537 \mathrm{e}-05 \quad 2.63419972669611 \mathrm{e}-07 \quad 5.30$

86: 4863:plaq $1160 \quad 0.5521634123414451 .20816256730995 \mathrm{e}-05 \quad 2.11092389021833 \mathrm{e}-08 \quad 2.04023761256621 \mathrm{e}-\mathrm{c}$ 87: 4928:rect $11600.3187373885435341 .66576568870565 \mathrm{e}-05 \quad 3.72092081884826 \mathrm{e}-08 \quad 4.30710452177097 \mathrm{e}-0$ 88: 5193:wline $4680-5.85079310776415 e-050.0004296531370274448 .6616078517946 e-056.166665777628$ 89: $776:$ tob3.66 10400

90: $2939: \mathrm{hmc} 11000.4464513907892031 .009022611732160 .7570807056$ 
91: $550: \operatorname{pbp} 2200$

92: $5559: 1.6999999999999999 \mathrm{e}-03 \quad 0.01464797118503897 .02992639329184 \mathrm{e}-05 \quad 2.49839023432037 \mathrm{e}-07 \quad 6.513$ 93: 5353:1.7000000000000001e-02 $0.055843937201486 \quad 3.41274614642902 \mathrm{e}-05 \quad 6.99127349016277 \mathrm{e}-081.574$ 94: 4930:plaq $1100 \quad 0.562063266452948$ 9.54571232953905e-06 $1.46224408874891 \mathrm{e}-08 \quad 1.08989055497663 \mathrm{e}-($ 95: 4797:rect $1100 \quad 0.33108483526125 \quad 1.38076217709706 \mathrm{e}-05 \quad 2.70318219895506 \mathrm{e}-08 \quad 2.2664541982793 \mathrm{e}-09$ 96: 5146:wline $4400-0.0004294933853008430 .0004288169540099819 .23833354106436 \mathrm{e}-05 \quad 6.10507488220$ 97: $777:$ :t0b3.76 10400

98: $3270:$ hmc $11200.284600816125053 \quad 0.970745276230174 \quad 0.790207479166667$

99: $552: \mathrm{pbp} 2220$

$100: 5537: 1.3899999999999999 e-02 \quad 0.0388090725947052 \quad 3.02710031244856 e-05 \quad 5.77683667353935 e-08 \quad 1.03$ 101: 5469:1.3900000000000000e-03 $0.007430376400614696 .73865165844095 \mathrm{e}-052.76252354944837 \mathrm{e}-075$. 102: 4935:plaq $1120 \quad 0.577028650533484 \quad 7.91657845685129 \mathrm{e}-06 \quad 1.28375358568775 \mathrm{e}-08 \quad 8.31155587334085 \mathrm{e}$ 103: 4922:rect $1120 \quad 0.3499319571377551 .17098243010848 \mathrm{e}-05 \quad 2.50199260796385 \mathrm{e}-081.71698302383937 \mathrm{e}$ 104: 5203:wline $4480-0.0003696826222252130 .0004367727921867868 .38439805926168$ e-05 5.6343859476

\section{P4RHMC therm 600}

1: $776: p 4 b 3.4610600$

2: $3158:$ hmc $11902.22294960984301 \quad 1.01127574711314 \quad 0.540457734186441$

3: $559:$ pbp 2380

4: $5264: 3.1300000000000001 \mathrm{e}-02 \quad 0.1548920462212090 .0001638325242474461 .40587650831366 \mathrm{e}-062.5631$

5: $5354: 3.1300000000000000 \mathrm{e}-03 \quad 0.0728358361297706 \quad 0.0002566043957632542 .57651128555722 \mathrm{e}-06 \quad 6.168$

6: 4888:plaq $1190 \quad 0.524123076177381 \quad 3.40661130359398 \mathrm{e}-05 \quad 1.03071516845062 \mathrm{e}-07 \quad 1.49441517804886 \mathrm{e}-0$

$7: 4809$ :rect $1190 \quad 0.28407693333504 \quad 4.76820402427467 \mathrm{e}-05 \quad 1.84657875755109 \mathrm{e}-07 \quad 2.7411261763835 \mathrm{e}-08$

8: 5033:wline $47600.004943115740757880 .000634290902381883 \quad 7.4297680581571 \mathrm{e}-05 \quad 6.70457555074424 \mathrm{e}$ 9: $779: \mathrm{p} 4 \mathrm{~b} 3.4910600$

10: $3199: \mathrm{hmc} 27101.871938920711891 .06892256652358 \quad 0.564468675303318$

11: $557: \mathrm{pbp} 5420$

12: $5491: 2.8999999999999998 \mathrm{e}-03 \quad 0.05150733397218730 .000137387458683526 \quad 3.09445918904089 \mathrm{e}-06 \quad 4.24$

13: $5326: 2.9000000000000001 \mathrm{e}-02 \quad 0.1308453075740148 .10595025361931 \mathrm{e}-051.25391003293854 \mathrm{e}-061.278$ 14: 4846:plaq $2700 \quad 0.5310927105670851 .4927094420713 \mathrm{e}-05 \quad 7.95437287220186 \mathrm{e}-08 \quad 4.38505338223842 \mathrm{e}-0$ 15: 4824 :rect $2700 \quad 0.292693903848865 \quad 2.061522646518 \mathrm{e}-05 \quad 1.41532736894146 \mathrm{e}-07 \quad 7.62179188955521 \mathrm{e}-09$ 16: 5083:wline $108800.008948006599968060 .000351058994032178 .42881677005643 e-05 \quad 3.71989102913019$ 17: $772:$ p4b3.51 10600

18: $3265: \mathrm{hmc} 4110 \quad 1.67182688217686 \quad 0.975899656347828 \quad 0.571444513937322$

19: $556: \mathrm{pbp} 8200$

20: $5477: 2.5899999999999999 \mathrm{e}-02 \quad 0.113321310865038 \quad 5.89261916049281 \mathrm{e}-05 \quad 1.10647747123483 \mathrm{e}-068.835$ 21: $5456: 2.5899999999999999 \mathrm{e}-03 \quad 0.0404760565707320 .0001097251657325293 .47392072697767 \mathrm{e}-06 \quad 2.899$ 22: $4900:$ plaq $4100 \quad 0.5353209845041621 .14812751710458 \mathrm{e}-05 \quad 7.95625692265719 \mathrm{e}-08 \quad 3.90903109404567 \mathrm{e}-\mathrm{c}$ 23: 4865 : rect $4100 \quad 0.2978942132185181 .59763816497404 \mathrm{e}-05 \quad 1.4320479361626 \mathrm{e}-07 \quad 7.79127539133138 \mathrm{e}-0 \mathrm{~s}$ 24: 4966:wline $163600.01121462775319420 .000286421346863939 .0755557515981 \mathrm{e}-053.10712264875198 \mathrm{e}$ 25: $775: \mathrm{p} 4 \mathrm{~b} 3.5410600$

26: $3258: \mathrm{hmc} 40801.30863746833297 \quad 0.9850757547232110 .608099288679309$

27: $561: \mathrm{pbp} 8160$

28: $5478: 2.3999999999999998 \mathrm{e}-03 \quad 0.0218540603315167 \quad 0.000141898198067623 \quad 6.71029081570833 e-06 \quad 4.84$ 29: 5457:2.4000000000000000e-02 $0.0934676676498867 \quad 7.31464669200508 \mathrm{e}-05 \quad 1.78951599556486 \mathrm{e}-06 \quad 1.26$ 30: 4862:plaq $40800.541502004991791 \quad 1.2302315524359 \mathrm{e}-05 \quad 8.74297488418643 \mathrm{e}-08 \quad 4.17966750935711 \mathrm{e}-0$ 31: 4909 : rect $4070 \quad 0.3055994191516341 .73260674002148 \mathrm{e}-05 \quad 1.62802831815193 \mathrm{e}-07 \quad 7.92793530877286 \mathrm{e}-\mathrm{c}$ 32: $4954:$ wline $16240 \quad 0.02279662770095520 .0003183566247771040 .000100248855187693 .54072576011835$ 33: $778: p 4 b 3.57 \quad 10600$ 
34: $3190: \mathrm{hmc} 34601.065930479765051 .036161959320920 .649469491262238$

35: $563: \mathrm{pbp} 6920$

36: 5514:2.1199999999999999e-03 $0.01103252472929227 .80319285730651 \mathrm{e}-05 \quad 1.67968032572671 \mathrm{e}-06 \quad 1.300$ 37: 5369:2.1200000000000000e-02 $0.0742189951418474 \quad 7.5580632730274 \mathrm{e}-05 \quad 1.58866348197691 \mathrm{e}-06 \quad 1.123$ 38: 4924 : plaq $3480 \quad 0.547275848595447 \quad 1.21761023272234 \mathrm{e}-05 \quad 7.82166026503599 \mathrm{e}-08 \quad 3.57538493750075 \mathrm{e}-\mathrm{c}$ 39: 4905:rect $34800.3128083266298411 .72441932560048 \mathrm{e}-05 \quad 1.44745660563294 \mathrm{e}-07 \quad 7.18568462445211 \mathrm{e}-\mathrm{c}$ 40: 4900:wline $138800.03422262986484010 .0003846097047934390 .000113160607330335 .1429811985923 \mathrm{e}$ 41: $772:$ p4b3.60 10600

42: $3264: \mathrm{hmc} 5070 \quad 0.9412798771439821 .00476670659248 \quad 0.679367327096195$

43: $604: \mathrm{pbp} 10180$

44: $5480: 1.9199999999999998 \mathrm{e}-02 \quad 0.0614352701852741 \quad 3.40239383969032 \mathrm{e}-05 \quad 4.9704533664256 \mathrm{e}-07 \quad 3.492$ 45: 5445:1.9200000000000000e-03 $0.00714992860520512 \quad 2.28681649701268 \mathrm{e}-05 \quad 2.26271505442232 \mathrm{e}-07 \quad 2.68$ 46: 4928:plaq 50800.552517811638668 8.28110648898584e-06 $6.68215422366005 \mathrm{e}-08 \quad 2.27331009588835 \mathrm{e}-\mathrm{c}$ 47: 4875:rect $50800.3193099083517691 .14143024159161 \mathrm{e}-05 \quad 1.20701194300509 \mathrm{e}-07 \quad 4.25312768143368 \mathrm{e}-($ 48: 4937:wline 203200.0423748820159490 .0002743074083162350 .0001048193084043163 .12124889762836 49: $775: \mathrm{p} 4 \mathrm{~b} 3.6310600$

50: $3231:$ hmc $6660 \quad 0.895500012932011 \quad 1.032218721214840 .687868849067657$

51: $605: \mathrm{pbp} 13340$

52: $5575: 1.6999999999999999 \mathrm{e}-03 \quad 0.00539917141846998 \quad 1.17471397566525 \mathrm{e}-05 \quad 8.10734754001604 \mathrm{e}-08 \quad 1.1$ 53: 5418:1.7000000000000001e-02 $0.05039805425262862 .58095589107762 \mathrm{e}-05 \quad 3.92059913790508 \mathrm{e}-07 \quad 2.273$ 54: 4911:plaq $6650 \quad 0.557542330900055 \quad 7.93182527638662 \mathrm{e}-06 \quad 7.21184756927059 \mathrm{e}-08 \quad 2.50222465083704 \mathrm{e}-$ 55: 4928:rect $66500.3255648450357231 .13267268989033 e-05 \quad 1.36009057135099 e-07 \quad 5.15687623365988 e-c$ 56: 5053:wline $266000.05129902964817950 .000245824846808567 \quad 0.0001098264658061692 .8948963347460$ 57: $778: p 4 b 3.6610600$

58: $3232:$ hmc $5870 \quad 0.838427553726548 \quad 1.01053743221203 \quad 0.676223377958253$

59: $611: \mathrm{pbp} 11780$

60: 5557:1.6999999999999999e-03 $0.004922483098748496 .7910456039674 \mathrm{e}-06 \quad 2.33795342202328 \mathrm{e}-08 \quad 3.18$ 61: $5416: 1.7000000000000001 \mathrm{e}-02 \quad 0.04735066949407291 .92030546605142 \mathrm{e}-05 \quad 1.87525769671555 \mathrm{e}-07 \quad 1.19$ 62: 4953:plaq $5880 \quad 0.562288216728843 \quad 8.23841464785485 \mathrm{e}-06 \quad 6.93948223734013 \mathrm{e}-08 \quad 2.71669356287248 \mathrm{e}-($ 63: 4930 :rect $5890 \quad 0.3314656627746181 .19627300182969 \mathrm{e}-05 \quad 1.35485178489208 \mathrm{e}-07 \quad 5.25857057010844 \mathrm{e}-\mathrm{c}$ 64: 5014:wline 235600.05957303480120940 .0002557271966127030 .0001022872398158523 .06470689839419 65: $781: \mathrm{p} 4 \mathrm{~b} 3.6910600$

66: $3207: \mathrm{hmc} 7210 \quad 0.808342335637398 \quad 1.00423434660937 \quad 0.681003566071103$

67: $605: \mathrm{pbp} 14420$

68: 5489:1.5000000000000000e-03 $0.00405484443933713 \quad 3.78595978644393 e-06 \quad 9.13309264929983 e-092.1$ 69: 5575:1.4999999999999999e-02 $0.03974404109486491 .25472611019545 \mathrm{e}-05 \quad 9.98412705863928 \mathrm{e}-08 \quad 5.92$ 70: 4961 : plaq $7210 \quad 0.566911127894627 \quad 6.48633185884298 \mathrm{e}-06 \quad 6.10684578898172 \mathrm{e}-08 \quad 1.75757016596066 \mathrm{e}-\mathrm{c}$ 71: 4910:rect $7210 \quad 0.337247378670948 \quad 9.20685232240132 \mathrm{e}-06 \quad 1.14975558251806 \mathrm{e}-07 \quad 3.46666041495145 \mathrm{e}-\mathrm{c}$ 72: $5032:$ wline 287600.06749133131880240 .0002499709321598030 .0001123686034310772 .7596536788457 73: $779:$ p4b3.76 10600

74: 3243:hmc $7440 \quad 0.759692516980502 \quad 1.01992654006538 \quad 0.707457052539474$

75: $610:$ pbp 14920

76: 5593:1.3899999999999999e-02 $0.03356801764464336 .77975634397195 \mathrm{e}-06 \quad 2.98975277267507 \mathrm{e}-08$ 1.772 $77: 5448: 1.3900000000000000 \mathrm{e}-03 \quad 0.00337895583790045 \quad 1.22291716296018 \mathrm{e}-06 \quad 9.83691021068614 \mathrm{e}-10 \quad 1.3$ 78: 4862 :plaq $7450 \quad 0.5771154298123396 .10634090372435 \mathrm{e}-06 \quad 5.9354153896824 \mathrm{e}-08 \quad 1.57016119660907 \mathrm{e}-0$ 79: 4916:rect $7470 \quad 0.3500895292434318 .81572543217354 \mathrm{e}-06 \quad 1.16221935789806 \mathrm{e}-07 \quad 3.08280421793909 \mathrm{e}-\mathrm{c}$ 80: 5047 :wline $29880 \quad 0.08517390794963130 .000258778674634477 \quad 0.000119962175297622 \quad 3.3043748434118$ 81: $772:$ :0b3.60 10600

82: $3328:$ hmc 11700.7549079839261020 .9899022085509770 .685886038578948

83: $555:$ pbp 2340 
84: $5512: 1.9199999999999998 \mathrm{e}-02 \quad 0.0715177012510356 \quad 4.60119221603279 \mathrm{e}-05 \quad 1.01948565979044 \mathrm{e}-072.16$ 85: $5352: 1.9200000000000000 \mathrm{e}-03 \quad 0.02305822080430848 .3216422768765 \mathrm{e}-05 \quad 2.52900000041564 \mathrm{e}-07 \quad 5.795$ 86: 4852:plaq $1160 \quad 0.5521611192538891 .32531250831358 \mathrm{e}-05 \quad 1.98623512488529 \mathrm{e}-08 \quad 1.7395609273144 \mathrm{e}-0$ 87: 4925 : rect $1160 \quad 0.3187347001111631 .75903928968858 \mathrm{e}-05 \quad 3.33969259139894 \mathrm{e}-08 \quad 3.71443254671542 \mathrm{e}-\mathrm{c}$ 88: 5053:wline $46800.0001983409899005810 .000486498968360688 .8501858691312 \mathrm{e}-057.66739061713904$ 89: 778:t0b3.66 10600

90: $2959: \mathrm{hmc} 1100 \quad 0.472215010290683 \quad 0.992132014993572 \quad 0.743779972$

91: $550:$ pbp 2200

92: $5575: 1.6999999999999999 \mathrm{e}-03 \quad 0.0148447200167276 \quad 5.82157639708388 \mathrm{e}-05 \quad 7.08778183949784 \mathrm{e}-08 \quad 3.50$ 93: $5450: 1.7000000000000001 \mathrm{e}-02 \quad 0.0559149553774833 \quad 3.12751730407965 \mathrm{e}-05 \quad 3.69535387191068 \mathrm{e}-088.86$ 94: 4814:plaq $1100 \quad 0.56205674768016 \quad 1.04671028252924 \mathrm{e}-05 \quad 1.3936936849035 \mathrm{e}-08 \quad 1.18948691626677 \mathrm{e}-09$ 95: 4792 :rect $1100 \quad 0.331076732527526 \quad 1.56280331435729 \mathrm{e}-05 \quad 2.5662624559275 \mathrm{e}-08 \quad 2.6829536119062 \mathrm{e}-09$ 96: 5114:wline $4400-7.84211288898962 \mathrm{e}-050.0005322704520911029 .988336628208 \mathrm{e}-05 \quad 7.55511623073948$ 97: 779:t0b3.76 10600

98: $3309:$ hmc $1120 \quad 0.275627557957297 \quad 0.975483006085166 \quad 0.792153459807693$

99: $552:$ pbp 2220

100: $5566: 1.3899999999999999 \mathrm{e}-02 \quad 0.0389282962804442 \quad 2.14701783521565 \mathrm{e}-05 \quad 1.65185868087344 \mathrm{e}-084.18$ 101: 5447:1.3900000000000000e-03 $0.00769094219218009 \quad 5.40761004251844 \mathrm{e}-05 \quad 9.95331105526766 \mathrm{e}-082$. 102: 4816:plaq $1120 \quad 0.577022292640905 \quad 8.8332077685005 \mathrm{e}-06 \quad 1.2403961869386 \mathrm{e}-08 \quad 9.75139051687879 \mathrm{e}-1$ 103: 4928:rect $11200.3499221264373821 .26995637649495 \mathrm{e}-05 \quad 2.31352508742391 \mathrm{e}-08 \quad 1.77969854308712 \mathrm{e}$ 104: 5197:wline $4480-0.0003343579171525890 .0005420868959502298 .67124990884498$ e-05 6.1541956346

\section{P4RHMC therm 800}

1: $778: \mathrm{p} 4 \mathrm{~b} 3.4610800$

2: $3230:$ hmc $11902.21781084247611 \quad 0.867940109098179 \quad 0.536787421820512$

3: $559:$ pbp 2380

4: $5271: 3.1300000000000001 \mathrm{e}-02 \quad 0.155408793157475 \quad 0.0001392622683695895 .88049095640476 \mathrm{e}-07 \quad 1.3931$

5: $5315: 3.1300000000000000 \mathrm{e}-03 \quad 0.0735757950623503 \quad 0.00027197839948154 \quad 1.56953023831456 \mathrm{e}-06 \quad 4.9344$ 6: 4892 :plaq $1190 \quad 0.524038465217624 \quad 3.00003429834583 e-05 \quad 6.78306351713623 e-08 \quad 9.28935290420653 e-05$ 7: 4896:rect $1190 \quad 0.283950046401501 \quad 4.16602329672697 \mathrm{e}-05 \quad 1.17492966244369 \mathrm{e}-07 \quad 1.69356007213047 \mathrm{e}-0$ 8: 5042 :wline $4760 \quad 0.005061165137087190 .000894117140535173 \quad 8.48535150540902 \mathrm{e}-05 \quad 9.04564521432219$ 9: $781: \mathrm{p} 4 \mathrm{~b} 3.4910800$

10: $3167: \mathrm{hmc} 27101.870471411221121 .07127044921677 \quad 0.564067090989529$

11: $557:$ pbp 5420

12: $5442: 2.8999999999999998 \mathrm{e}-03 \quad 0.0517580942407844 \quad 0.0001329674177096932 .49400932384138 \mathrm{e}-06 \quad 3.80$ 13: $5280: 2.9000000000000001 \mathrm{e}-02 \quad 0.1309948900884 \quad 7.87806291068086 \mathrm{e}-05 \quad 1.05446882098776 \mathrm{e}-06 \quad 1.14973$ 14: 4926:plaq $2700 \quad 0.531071851327161 \quad 1.48746692431534 \mathrm{e}-05 \quad 7.45936981732794 \mathrm{e}-08 \quad 4.12061093966989 \mathrm{e}-\mathrm{c}$ 15: 4915 : rect $2700 \quad 0.2926658888625642 .07100525288079 \mathrm{e}-05 \quad 1.33711380815553 \mathrm{e}-07 \quad 7.32960336464476 \mathrm{e}-\mathrm{c}$ 16: 5137 :wline 108800.008738137130105170 .0003718221633818288 .24459716506468 e-05 3.785689295095" 17: $774:$ p4b3.51 10800

18: $3163: \mathrm{hmc} 41101.67530791542705 \quad 0.969723146091340 .570513453151058$

19: $556: \mathrm{pbp} 8200$

$20: 5435: 2.5899999999999999 \mathrm{e}-02 \quad 0.113320186263214 \quad 6.1891167959934 \mathrm{e}-05 \quad 1.15397511074663 \mathrm{e}-06 \quad 9.28968$ 21: $5497: 2.5899999999999999 \mathrm{e}-03 \quad 0.0405068081231172 \quad 0.000113893155713957 \quad 3.53969147674958 \mathrm{e}-06 \quad 3.028$ 22: 4915 :plaq $41000.5353223275762951 .17598869436017 \mathrm{e}-05 \quad 7.91829720512821 \mathrm{e}-08 \quad 4.00993396201827 \mathrm{e}-\mathrm{c}$ 23: 4892 :rect $4100 \quad 0.297896517900871 \quad 1.64070171879962 \mathrm{e}-05 \quad 1.43064189839436 \mathrm{e}-07 \quad 7.9750276739727 \mathrm{e}-0$ 24: 5049:wline $163600.01112905734917050 .000295182037559183 \quad 9.08005597216452 \mathrm{e}-05 \quad 3.2135706039570$ 25: $777: p 4 b 3.5410800$

26: $3041:$ hmc $40801.30616120057779 \quad 0.994459025430051 \quad 0.610154013425$ 
27: $561: \mathrm{pbp} 8160$

28: $5495: 2.3999999999999998 \mathrm{e}-03 \quad 0.02170726328525650 .0001451186556955626 .61641455926368 \mathrm{e}-06 \quad 4.89$ 29: $5353: 2.4000000000000000 \mathrm{e}-02 \quad 0.09338876801053 \quad 7.51169790567769 \mathrm{e}-05 \quad 1.77839379782914 \mathrm{e}-06 \quad 1.29948$ 30: 4942:plaq $40800.541512854203649 \quad 1.24689335715679 \mathrm{e}-05 \quad 8.63689762598663 \mathrm{e}-08 \quad 4.20571080909673 \mathrm{e}-($ 31: 4913:rect $40700.3056153403939051 .76545543239328 \mathrm{e}-05 \quad 1.61451882508697 \mathrm{e}-07 \quad 8.06881506115567 \mathrm{e}-\mathrm{c}$ 32: 5052:wline $16240 \quad 0.02299601497458720 .0003238430053345859 .91687779499053 e-05 \quad 3.5867831647249$ 33: $780: p 4 b 3.5710800$

34: $3186: \mathrm{hmc} 34601.064533709805031 .02825688931626 \quad 0.644938696804511$

35: $563: \mathrm{pbp} 6920$

36: 5418:2.1199999999999999e-03 $0.010971485656902 \quad 8.0915527078029 \mathrm{e}-05 \quad 1.68048402022935 \mathrm{e}-06 \quad 1.3924$ 37: 5425:2.1200000000000000e-02 $0.0741420189587355 \quad 7.66612256136533 \mathrm{e}-05 \quad 1.51843988879985 \mathrm{e}-06 \quad 1.16$ 38: 4911 : plaq $34800.547283430807907 \quad 1.25532470273144 \mathrm{e}-05 \quad 7.81126463072465 \mathrm{e}-08 \quad 3.78779042207874 \mathrm{e}-($ 39: 4911 : rect $34800.3128193787945491 .78000300413668 \mathrm{e}-05 \quad 1.43915034264334 \mathrm{e}-07 \quad 7.64327655023998 \mathrm{e}-\mathrm{c}$ 40: 4968:wline 138800.03460229001936490 .0004004070550662710 .0001140228046730425 .4481045516329 41: $774:$ p4b3.60 10800

42: $3166: \mathrm{hmc} 5070 \quad 0.945305360865075 \quad 1.00327353703354 \quad 0.66434940330679$

43: $604: \mathrm{pbp} 10180$

44: $5494: 1.9199999999999998 \mathrm{e}-02 \quad 0.0614726412691523 \quad 3.4182375518898 \mathrm{e}-05 \quad 4.78355222131696 \mathrm{e}-07 \quad 3.530$ 45: $5461: 1.9200000000000000 \mathrm{e}-03 \quad 0.00716521626947997 \quad 2.34986176126627 \mathrm{e}-05 \quad 2.28164742721211 \mathrm{e}-07 \quad 2.7$ 46: 4861 :plaq $50800.552510974161013 \quad 8.3830979148709 \mathrm{e}-06 \quad 6.54993181354121 \mathrm{e}-08 \quad 2.26062860982815 \mathrm{e}-0$ 47: 4950:rect $50800.3192998859077151 .15357263291359 \mathrm{e}-05 \quad 1.18716980056695 \mathrm{e}-07 \quad 4.24660878496753 \mathrm{e}-\mathrm{c}$ 48: 5005:wline $203200.04233680892051840 .0002829650847336590 .000104918119000676 \quad 3.2243963782097$ 49: $777:$ p4b3.63 10800

50: $3274:$ hmc $6660 \quad 0.894952460889569 \quad 1.02628036317176 \quad 0.677215908762799$

51: $605: \mathrm{pbp} 13340$

52: 5515:1.6999999999999999e-03 $0.0054033293234678 \quad 1.20810381152784 \mathrm{e}-05 \quad 8.29124265825425 \mathrm{e}-08 \quad 1.15$ 53: 5343:1.7000000000000001e-02 $0.05041048056389892 .6390633880201 \mathrm{e}-05 \quad 3.96513643950917 \mathrm{e}-072.325$ 54: 4892:plaq $6650 \quad 0.557536357795685 \quad 7.99377621507033 \mathrm{e}-06 \quad 7.09105874633344 \mathrm{e}-08 \quad 2.5181041259698 \mathrm{e}-0$ 55: 4856:rect $6650 \quad 0.3255571393616421 .14441681440951 \mathrm{e}-05 \quad 1.33985770185281 \mathrm{e}-07 \quad 5.2499219461191 \mathrm{e}-0$ 56: $5017:$ wline $26600 \quad 0.05132944106303240 .0002513243375408670 .0001098679500184572 .96585558648388$ 57: $780:$ p4b3.66 10800

58: $3234: \mathrm{hmc} 5870 \quad 0.839171053006703 \quad 1.00550717810277 \quad 0.670785975607494$

59: $611: \mathrm{pbp} 11780$

60: $5644: 1.6999999999999999 \mathrm{e}-03 \quad 0.00492636579526888 \quad 6.98232449801878 \mathrm{e}-06 \quad 2.37643961454108 \mathrm{e}-08 \quad 3.2$ 61: $5430: 1.7000000000000001 \mathrm{e}-02 \quad 0.04736270560936681 .95787974575073 \mathrm{e}-05 \quad 1.87500910247333 \mathrm{e}-07 \quad 1.205$ 62: 4905:plaq $5880 \quad 0.562285620787328 .35468086265378 \mathrm{e}-06 \quad 6.86743909134604 \mathrm{e}-08 \quad 2.76030992287679 \mathrm{e}-0$ 63: 4927:rect $5890 \quad 0.331461834020731 \quad 1.21357652528972 \mathrm{e}-05 \quad 1.33918989322956 \mathrm{e}-07 \quad 5.31397809958332 \mathrm{e}-\mathrm{c}$ 64: 4978:wline 235600.05943312163590330 .0002597805537603120 .0001020212208486573 .1486326320817 ! 65: $783: \mathrm{p} 4 \mathrm{~b} 3.6910800$

66: $3225:$ hmc $7210 \quad 0.813191037499113 \quad 1.003343740851250 .674951337992198$

67: $605: \mathrm{pbp} 14420$

68: 5470:1.5000000000000000e-03 $0.004053942369968913 .88292650254013 \mathrm{e}-06 \quad 9.32040894060642 \mathrm{e}-092.1$ 69: 5449:1.4999999999999999e-02 $0.0397378461535351 .27307035550035 \mathrm{e}-05 \quad 9.9672121382823 \mathrm{e}-08$ 6.0949" $70: 4908:$ plaq $7210 \quad 0.566910036482541 \quad 6.60156769146781 \mathrm{e}-06 \quad 6.12372192254334 \mathrm{e}-08 \quad 1.79121485067359 \mathrm{e}-($ 71: 4962 : rect $7210 \quad 0.337246856644477 \quad 9.38095581936067 \mathrm{e}-06 \quad 1.15543664281477 \mathrm{e}-07 \quad 3.54896972395498 \mathrm{e}-\mathrm{c}$ 72: $5051:$ wline 287600.06760377818725370 .0002544664542470890 .0001128055691285992 .8080179388459 73: $781: \mathrm{p} 4 \mathrm{~b} 3.7610800$

74: $3254:$ hmc $7440 \quad 0.758930000980699 \quad 1.01597801280567 \quad 0.698645517977411$

75: $610:$ pbp 14920

$76: 5484: 1.3899999999999999 \mathrm{e}-02 \quad 0.03356511850031316 .82058384064985 \mathrm{e}-06$ 
77: 5386:1.3900000000000000e-03 $0.00337832234046011 \quad 1.2166536045267 \mathrm{e}-06 \quad 9.44238754220586 \mathrm{e}-10 \quad 1.388$ 78: 4965 : plaq $7450 \quad 0.577113509564914 \quad 6.18368935378767 \mathrm{e}-06 \quad 5.87415479966318 \mathrm{e}-08 \quad 1.58245315129788 \mathrm{e}-$ 79: 4965 :rect $7470 \quad 0.3500868874753698 .91259766027726 e-06 \quad 1.14934275280953 e-07 \quad 3.08983808758973 e-c$ 80: 5060:wline $298800.08516027418148720 .000264726967672694 \quad 0.0001204039658917383 .3817468929957$ 81: $774:$ :t0b3.60 10800

82: $3266:$ hmc $1170 \quad 0.756431735824422 \quad 0.961739710961271 \quad 0.679104231513513$

83: $555:$ pbp 2340

84: 5549:1.9199999999999998e-02 $0.07145371358423865 .86759899004171 \mathrm{e}-051.07526175435558 \mathrm{e}-072.86$ 85: $5368: 1.9200000000000000 \mathrm{e}-03 \quad 0.02292821285416440 .0001047308873584652 .57904276183886 \mathrm{e}-07 \quad 7.92$ 86: 4860 : plaq $1160 \quad 0.552156706277024 \quad 1.69229221509736 \mathrm{e}-05 \quad 2.08904262838989 \mathrm{e}-08 \quad 2.1916847320806 \mathrm{e}-0$ 87: 4920 :rect $1160 \quad 0.318731056331087 \quad 2.36693777950233 e-05 \quad 3.65965625716589 e-08 \quad 5.00006823729917 e-c$ 88: 5098:wline $46800.0008559439008826320 .0005651608337094629 .36332521868223 e-051.0230213129258$ 89: $780:$ tob3.66 10800

90: $2916: \mathrm{hmc} 1100 \quad 0.4800881607355931 .020475164595720 .754267893$

91: $550:$ pbp 2200

92: 5579:1.6999999999999999e-03 $0.01476978278450248 .54798286715396 \mathrm{e}-05 \quad 1.25263460222821 \mathrm{e}-07 \quad 4.86$ 93: 5439:1.7000000000000001e-02 $0.0558903460294667 \quad 4.39178105538669 \mathrm{e}-05 \quad 4.46800813194992 \mathrm{e}-08$ 1.25 94: 4900:plaq $1100 \quad 0.562057450615681 \quad 1.08036243906692 \mathrm{e}-05 \quad 1.25685342189374 \mathrm{e}-08 \quad 1.34990073557834 \mathrm{e}-\mathrm{c}$ 95: 4865 : rect $1100 \quad 0.331079097816896 \quad 1.53020358772446 \mathrm{e}-05 \quad 2.0809104953982 \mathrm{e}-08 \quad 2.38688471549181 \mathrm{e}-0$ s 96: 5129:wline $4400-0.000678346833245390 .0006876201049942779 .26801363174267 e-058.866189118906$ 97: $781:$ tob3.76 10800

98: $3147:$ hmc $1120 \quad 0.280593843614696 \quad 0.975932560807746 \quad 0.791467444375$

99: $552:$ pbp 2220

100: $5560: 1.3899999999999999 \mathrm{e}-02 \quad 0.03892174781848742 .72555400581201 \mathrm{e}-05 \quad 1.66928581339094 \mathrm{e}-08 \quad 5.90$ 101: 5406:1.3900000000000000e-03 $0.0078657321121296 \quad 6.44060606035926 \mathrm{e}-05 \quad 7.25715226849814 \mathrm{e}-082.6$ 102: $4879: \mathrm{plaq} 1120 \quad 0.577021113695687 \quad 1.21818458380594 \mathrm{e}-05 \quad 1.25733503816838 \mathrm{e}-08 \quad 1.30045118203124 \mathrm{e}$ 103: 4850:rect $1120 \quad 0.349922064624638 \quad 1.66668062256811 \mathrm{e}-05 \quad 2.32677506550177 \mathrm{e}-082.1625057993602 \mathrm{e}-($ 104: 5150:wline $4480-0.0009081621952661790 .000729040855488898 .82477888613217 e-057.19158385069$

\section{Diagnostic Results}




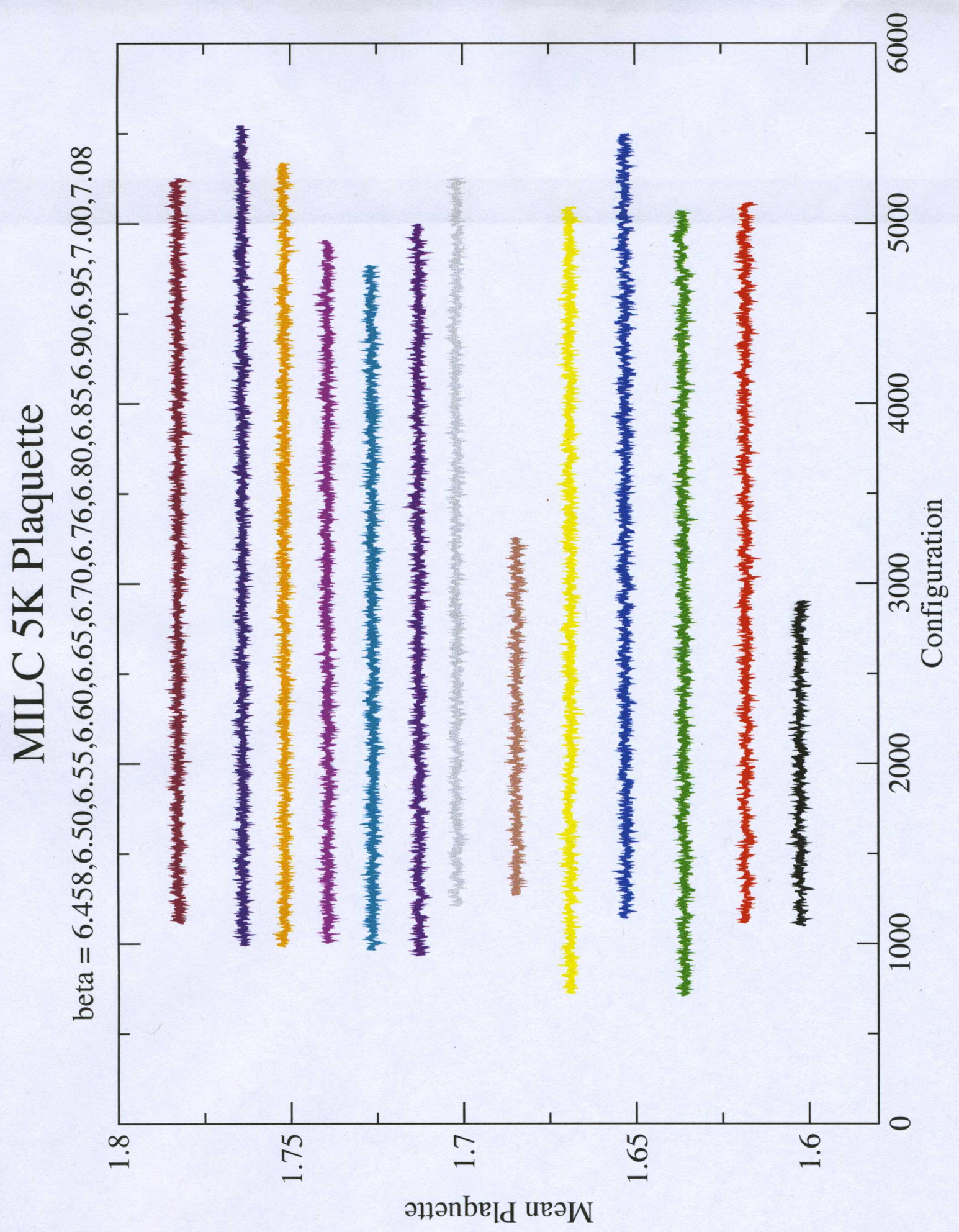




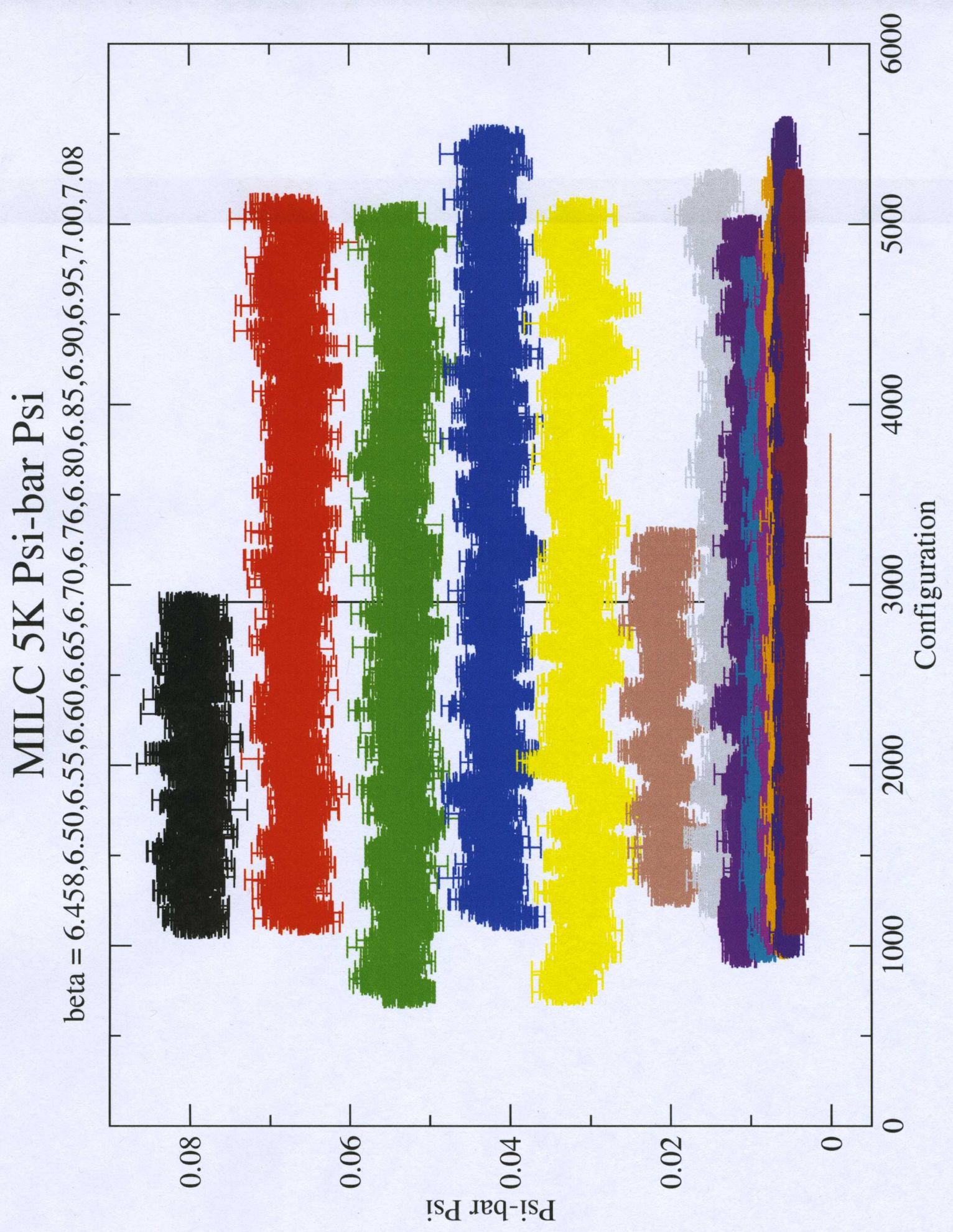




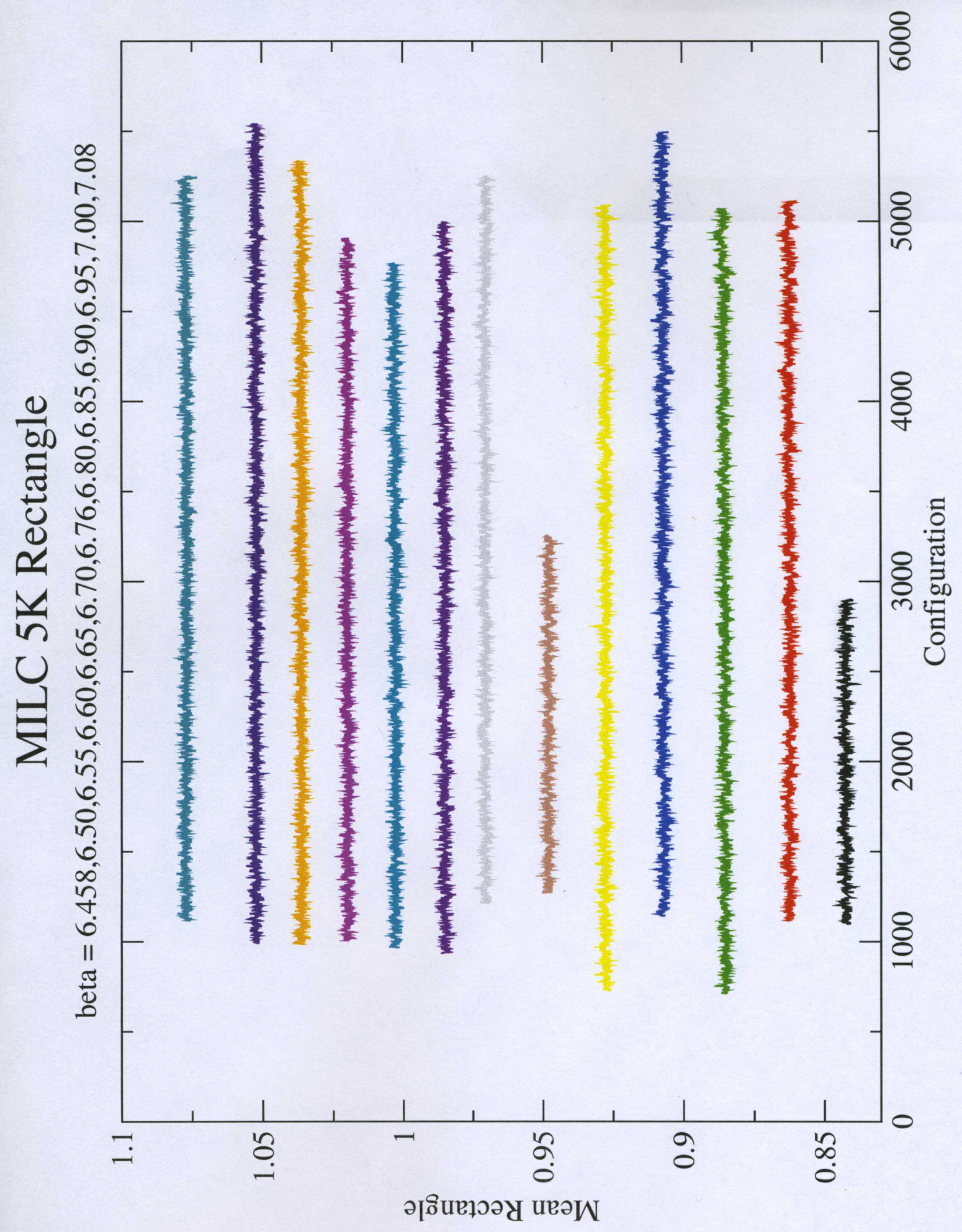




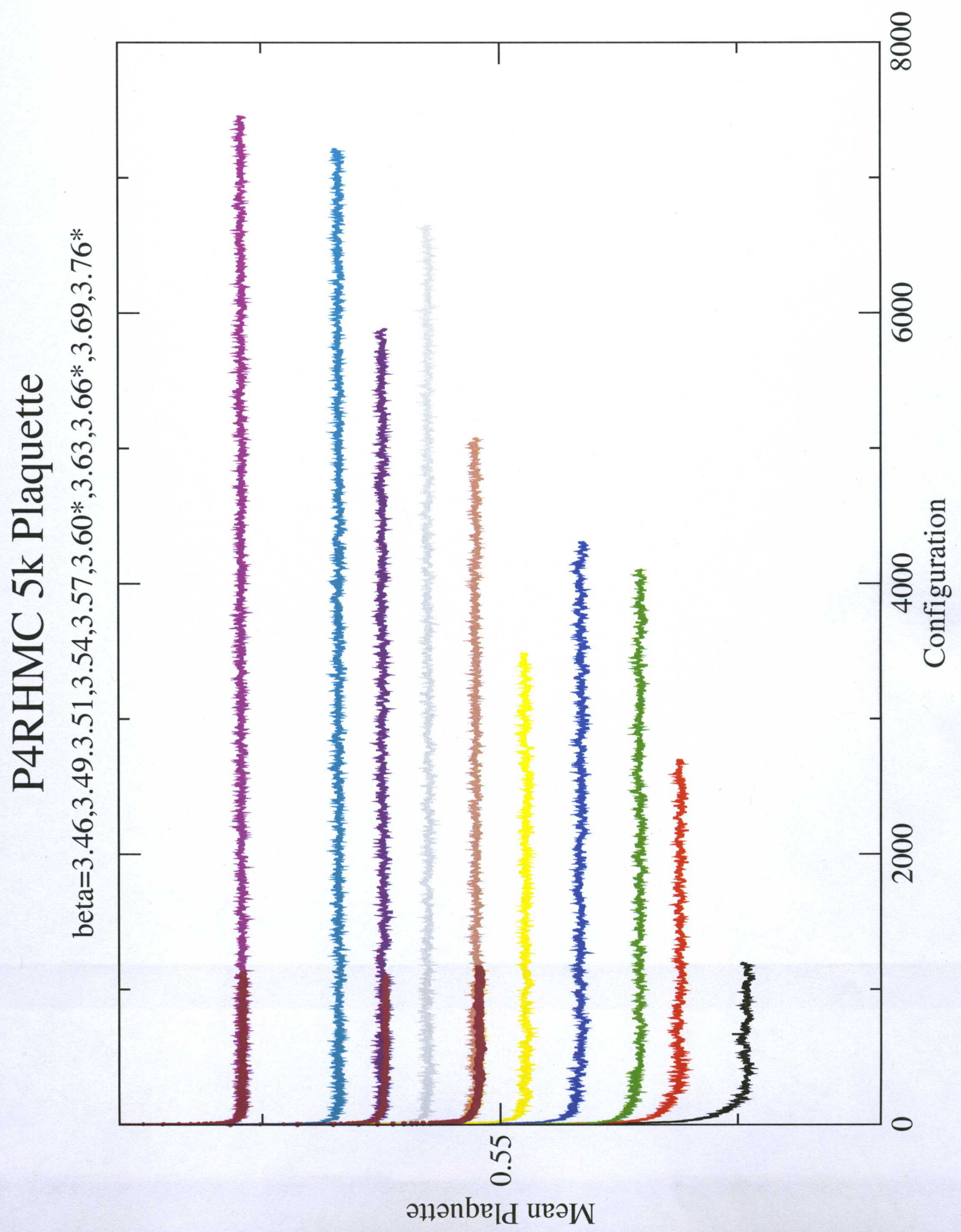




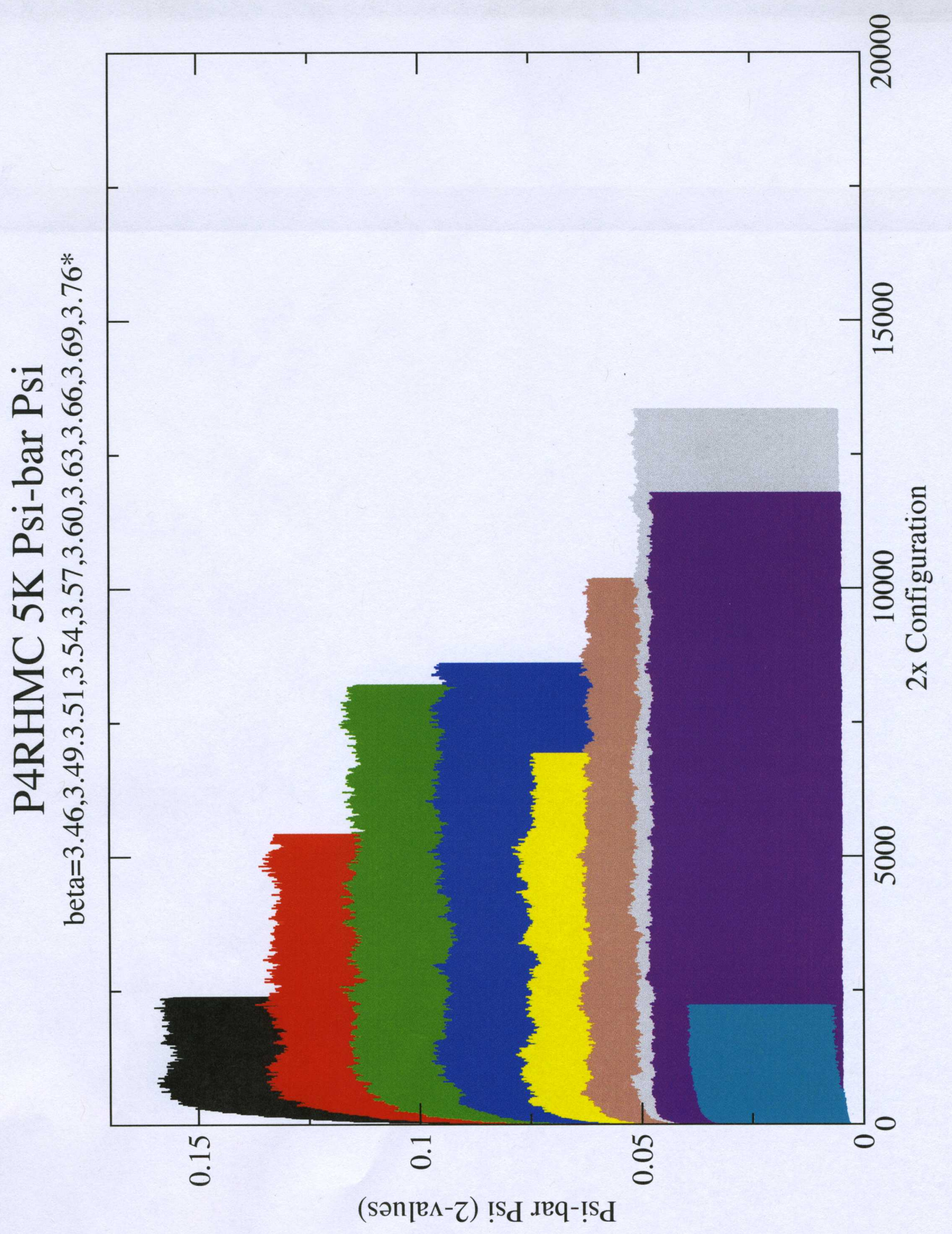




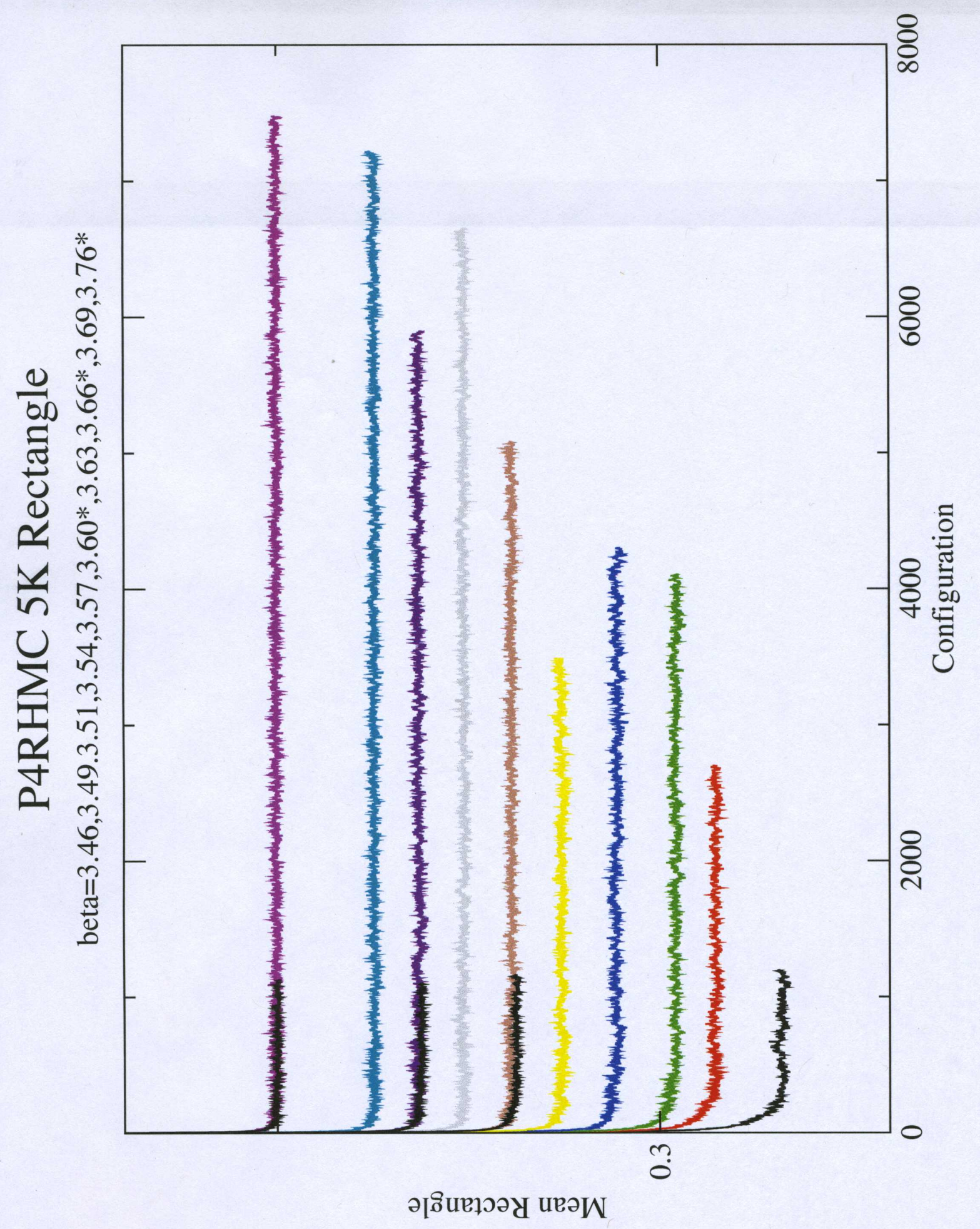




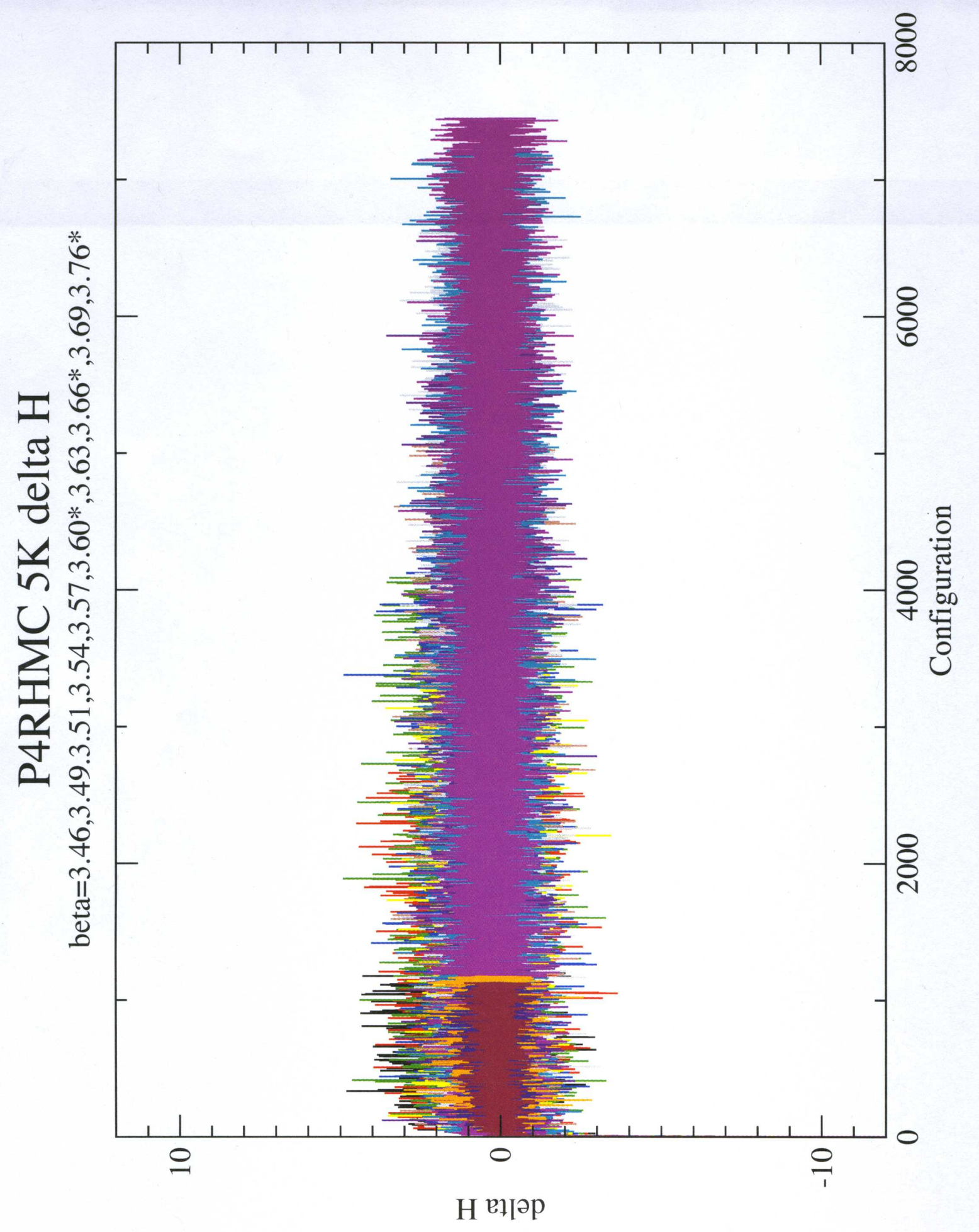

